\title{
Mutations of Fake Weighted Projective Spaces
}

\author{
Tom Coates Samuel Gonshaw Alexander Kasprzyk Navid Nabijou \\ Department of Mathematics \\ Imperial College London \\ London, SW7 2AZ, UK \\ t.coates@imperial.ac.uk \\ samuel.gonshaw10@imperial.ac.uk \\ a.m.kasprzyk@imperial.ac.uk \\ navid.nabijou09@imperial.ac.uk
}

Submitted: Apr 15, 2014; Accepted: Oct 7, 2014; Published: Oct 16, 2014

Mathematics Subject Classifications: 52B20 (Primary); 14J33, 14J45 (Secondary)

\begin{abstract}
We characterise mutations between fake weighted projective spaces, and give explicit formulas for how the weights and multiplicity change under mutation. In particular, we prove that multiplicity-preserving mutations between fake weighted projective spaces are mutations over edges of the corresponding simplices. As an application, we analyse the canonical and terminal fake weighted projective spaces of maximal degree.
\end{abstract}

Keywords: Lattice polytopes; mutations; cluster transformations; mirror symmetry; Fano varieties; canonical singularities; terminal singularities; projective space

\section{Introduction}

In this paper we analyse mutations between fake weighted projective spaces; equivalently we analyse mutations between lattice simplices. Mutations arise naturally when considering mirror symmetry for Fano manifolds. A Fano manifold $X$ is expected to correspond under mirror symmetry to a Laurent polynomial $[3,5,6,7,10,11,14,18]$. In general there will be many different Laurent polynomials which correspond to a given Fano manifold, and it is expected that these Laurent polynomials are related via birational transformations analogous to cluster transformations $[1,15,16,17]$. These cluster-style transformations act on Newton polytopes via mutations; see Definition 5 below. A mutation between Newton polytopes can be thought of as the tropicalisation of the corresponding cluster-type transformation. Ilten [20] has shown that if two lattice polytopes $P$ and $Q$ 
are related by mutation then the corresponding toric varieties $X_{P}$ and $X_{Q}$ are deformation equivalent: there exists a flat family $\mathcal{X} \rightarrow \mathbb{P}^{1}$ such that $\mathcal{X}_{0} \cong X_{P}$ and $\mathcal{X}_{\infty} \cong X_{Q}$. Mutations are thus expected to form the one-skeleton of the tropicalisation of the Hilbert scheme. Our understanding of this one-skeleton is rudimentary but improving $[1,2]$; in this paper we conduct the first systematic analysis in higher dimensions.

The notion of mutation raises many new and interesting combinatorial questions: for example, how can polytopes be classified up to mutation, and what properties of polytopes are mutation-invariant? Here we begin to address these questions by analysing the behaviour of lattice simplicies under mutation. In two dimensions, Akhtar and Kasprzyk determined how the weights of a fake weighted projective plane, i.e. the weights of a lattice triangle, change under mutation, and showed that mutations between fake weighted projective planes are multiplicity-preserving [2]. We show below that the situation in higher dimensions is different. We give an explicit formula (Theorem 12) for how the weights of a fake weighted projective space, i.e. the weights of a lattice simplex, change under mutation, and derive a strong necessary condition (Theorem 15) for a mutation to preserve multiplicity. In $\S \S 7-8$ we apply our results to the study of fake weighted projective spaces of high degree with canonical and terminal singularities.

\section{Fake Weighted Projective Space}

We begin by recalling some standard definitions. Throughout let $N \cong \mathbb{Z}^{n}$ denote a lattice of rank $n$ with dual lattice $M:=\operatorname{Hom}(N, \mathbb{Z})$. From the toric viewpoint $N$ corresponds to the lattice of one-parameter subgroups and $M$ corresponds to the lattice of characters. For an introduction to toric geometry see [13].

Definition 1. A convex lattice polytope $P \subset N_{\mathbb{Q}}:=N \otimes_{\mathbb{Z}} \mathbb{Q}$ is said to be Fano if:

1. $P$ is of maximum dimension, that is $\operatorname{dim} P=n$;

2. the origin is contained in the strict interior of $P$, that is $\mathbf{0} \in \operatorname{int}(P)$;

3. the vertices vert $(P)$ of $P$ are primitive lattice points.

In addition to being compelling combinatorial objects, Fano polytopes are in bijective correspondence with toric Fano varieties: see [24] for an overview. The complete fan in $N$ generated by the faces of $P$, which we call the spanning fan of $P$, corresponds to a toric Fano variety $X_{P}$, that is, to a (possibly singular) projective toric variety with ample anticanonical divisor $-K_{X}$. Two Fano polytopes $P$ and $P^{\prime}$ give isomorphic varieties $X_{P} \cong X_{P^{\prime}}$ if and only if there exists a change of basis of the underlying lattice $N$ sending $P$ to $P^{\prime}$. Thus we regard $P$ as being defined only up to $\mathrm{GL}_{n}(\mathbb{Z})$-equivalence.

Definition 2. Let $P:=\operatorname{conv}\left\{v_{0}, \ldots, v_{n}\right\} \subset N_{\mathbb{Q}}$ be a Fano $n$-simplex. By Definition 1(2) there exists a unique choice of $n+1$ coprime positive integers $\lambda_{0}, \ldots, \lambda_{n} \in \mathbb{Z}_{>0}$ such that $\lambda_{0} v_{0}+\ldots+\lambda_{n} v_{n}=\mathbf{0}$. These are called the (reduced) weights of $P$. 
Definition 1(3) implies that any $n$ of the weights are also coprime, or in other words that the weights are well-formed. See $[19, \S 5]$ for details of the natural role that reduced and well-formed weights play in the study of weighted projective space.

Definition 3. Let $P \subset N_{\mathbb{Q}}$ be a Fano $n$-simplex and let $N^{\prime}:=v_{0} \cdot \mathbb{Z}+\ldots+v_{n} \cdot \mathbb{Z}$ be the sublattice in $N$ generated by the vertices of $P$. The rank-one $\mathbb{Q}$-factorial toric Fano variety $X_{P}$ given by the spanning fan of $P$ is $X_{P}=\mathbb{P}\left(\lambda_{0}, \ldots, \lambda_{n}\right) /\left(N / N^{\prime}\right)$, where the group $N / N^{\prime}$ acts freely in codimension one. We call $X_{P}$ a fake weighted projective space.

Fake weighted projective spaces have been studied in $[9,12,21]$. One important invariant is the multiplicity: the index of the sublattice $N^{\prime}$ in $N$, denoted by $\operatorname{mult}(P):=$ $\left[N: N^{\prime}\right]$. A fake weighted projective space is a weighted projective space if and only if $\operatorname{mult}(P)=1[8$, Proposition 2].

\section{Mutations}

We recall the definition of mutation, following [1, §3]. A primitive element $w \in M$ determines a surjective linear map $w: N \rightarrow \mathbb{Z}$ which extends naturally to a map $N_{\mathbb{Q}} \rightarrow \mathbb{Q}$. A point $v \in N_{\mathbb{Q}}$ is said to be at height $w(v)$. Given a subset $S \subset N_{\mathbb{Q}}$, if $w(v)=h$ for all $v \in S$ we say that $S$ lies at height $h$ and write $w(S)=h$. The hyperplane $H_{w, h}$ is defined to be the set of all points in $N_{\mathbb{Q}}$ at height $h$. For a convex lattice polytope $P \subset N_{\mathbb{Q}}$ we define $w_{h}(P):=\operatorname{conv}\left(H_{w, h} \cap P \cap N\right)$ to be the (possibly empty) convex hull of all lattice points in $P$ at height $h$. We set $h_{\min }:=\min \{w(v) \mid v \in P\}$ to be the minimum height occurring amongst the the points of $P$, and $h_{\max }$ to be the maximum height. Since $P$ is a lattice polytope, both $h_{\min }$ and $h_{\max }$ are integers. If $\mathbf{0} \in \operatorname{int}(P)$, and in particular if $P$ is Fano, then $h_{\min }<0$ and $h_{\max }>0$.

Definition 4. A factor of $P \subset N_{\mathbb{Q}}$ with respect to a primitive height function $w \in M$ is a lattice polytope $F \subset N_{\mathbb{Q}}$ such that:

1. $w(F)=0$;

2. for every integer $h$ with $h_{\min } \leqslant h<0$, there exists a (possibly empty) lattice polytope $G_{h} \subset N_{\mathbb{Q}}$ such that $H_{w, h} \cap \operatorname{vert}(P) \subseteq G_{h}+(-h) F \subseteq w_{h}(P)$.

Definition 5. The (combinatorial) mutation of $P \subset N_{\mathbb{Q}}$ with respect to the primitive height function $w \in M$ and a factor $F \subset N_{\mathbb{Q}}$ is the convex lattice polytope

$$
\operatorname{mut}_{w}(P, F):=\operatorname{conv}\left(\bigcup_{h=h_{\min }}^{-1} G_{h} \cup \bigcup_{h=0}^{h_{\max }}\left(w_{h}(P)+h F\right)\right) \subset N_{\mathbb{Q}} .
$$

Although this is not obvious from the definition, the mutation $\operatorname{mut}_{w}(P, F)$ is independent of the choice of the $G_{h}\left[1\right.$, Proposition 1]. Furthermore, $\operatorname{mut}_{w}(P, F)$ is a Fano polytope if and only if $P$ is a Fano polytope [1, Proposition 2]. More obviously, mutations are always invertible (if $Q:=\operatorname{mut}_{w}(P, F)$ then $P \cong \operatorname{mut}_{-w}(Q, F)$ ) [1, Lemma 2], and 
translating the factor results in an isomorphic mutation (i.e. $\operatorname{mut}_{w}(P, F) \cong \operatorname{mut}_{w}(P, F+v)$ for any $v \in N$ with $w(v)=0)$.

Mutations have a natural description as transformations of the dual polytope

$$
P^{\vee}:=\left\{u \in M_{\mathbb{Q}} \mid u(v) \geqslant-1 \text { for all } v \in P\right\} .
$$

A mutation induces a piecewise $\mathrm{GL}_{n}(\mathbb{Z})$-transformation $\varphi: u \mapsto u-u_{\min } w$ of $M_{\mathbb{Q}}$ such that $\left(\varphi\left(P^{\vee}\right)\right)^{\vee}=\operatorname{mut}_{w}(P, F)$; here $u_{\text {min }}:=\min \{u(v) \mid v \in F\}$. This is analogous to a cluster transformation. As a consequence $\left|k P^{\vee} \cap M\right|=\left|k Q^{\vee} \cap M\right|$ for any dilation $k \in \mathbb{Z}_{\geqslant 0}$, where $Q:=\operatorname{mut}_{w}(P, F)[1$, Proposition 4$]$; hence $\operatorname{Hilb}\left(X_{P},-K_{X_{P}}\right)=\operatorname{Hilb}\left(X_{Q},-K_{X_{Q}}\right)$ and $X_{P}$ and $X_{Q}$ have the same anticanonical degree.

Example 6. The weighted projective spaces $\mathbb{P}(1,1,1,3)$ and $\mathbb{P}(1,1,4,6)$ have the largest degree amongst all canonical ${ }^{1}$ toric Fano threefolds $[22]$ and amongst all Gorenstein canonical Fano threefolds [26]. They are related by a mutation [1, Example 7]. The simplex associated to $\mathbb{P}(1,1,1,3)$ is $P:=\operatorname{conv}\{(1,0,0),(0,1,0),(0,0,1),(-1,-1,-3)\} \subset N_{\mathbb{Q}}$. Setting $w=(-1,2,0) \in M$ gives $h_{\min }=-1$ and $h_{\max }=2$, with $w_{-1}(P)$ equal to the edge $\operatorname{conv}\{(-1,-1,-3),(1,0,0)\}$ and $w_{2}(P)$ given by the vertex $(0,1,0)$. The factor

$$
F:=\operatorname{conv}\{(0,0,0),(2,1,3)\}
$$

gives:

$$
\operatorname{mut}_{w}(P, F)=\operatorname{conv}\{(-1,-1,-3),(0,0,1),(0,1,0),(4,3,6)\}
$$

and this is the simplex associated with $\mathbb{P}(1,1,4,6)$. This mutation is illustrated in Figure 1.

\section{Mutations of $\boldsymbol{n}$-Simplices}

We begin by establishing some basic properties of mutations between simplices. Throughout this section we assume that $P$ is a Fano $n$-simplex, and that $w \in M$ and $F \subset N_{\mathbb{Q}}$ are (respectively) a primitive height function and a factor such that $Q:=\operatorname{mut}_{w}(P, F)$ is a simplex. In other words, we assume that there is a mutation from the fake weighted projective space $X$ associated with $P$ to the fake weighted projective space $Y$ associated with $Q$.

Lemma 7. Let $P$ be a Fano $n$-simplex, let $w \in M$ be a primitive height function, and let $F \subset N_{\mathbb{Q}}$ be a factor such that $Q:=\operatorname{mut}_{w}(P, F)$ is a simplex. Suppose that the mutation from $P$ to $Q$ is non-trivial, so that $P \neq Q$. Then $w_{h_{\max }}(P)$ is a vertex of $P$ and $F$ is a translation of $\frac{1}{\left|h_{\min }\right|} w_{h_{\min }}(P)$.

\footnotetext{
${ }^{1}$ See $\S 6$ below for a discussion of canonical singularities.
} 


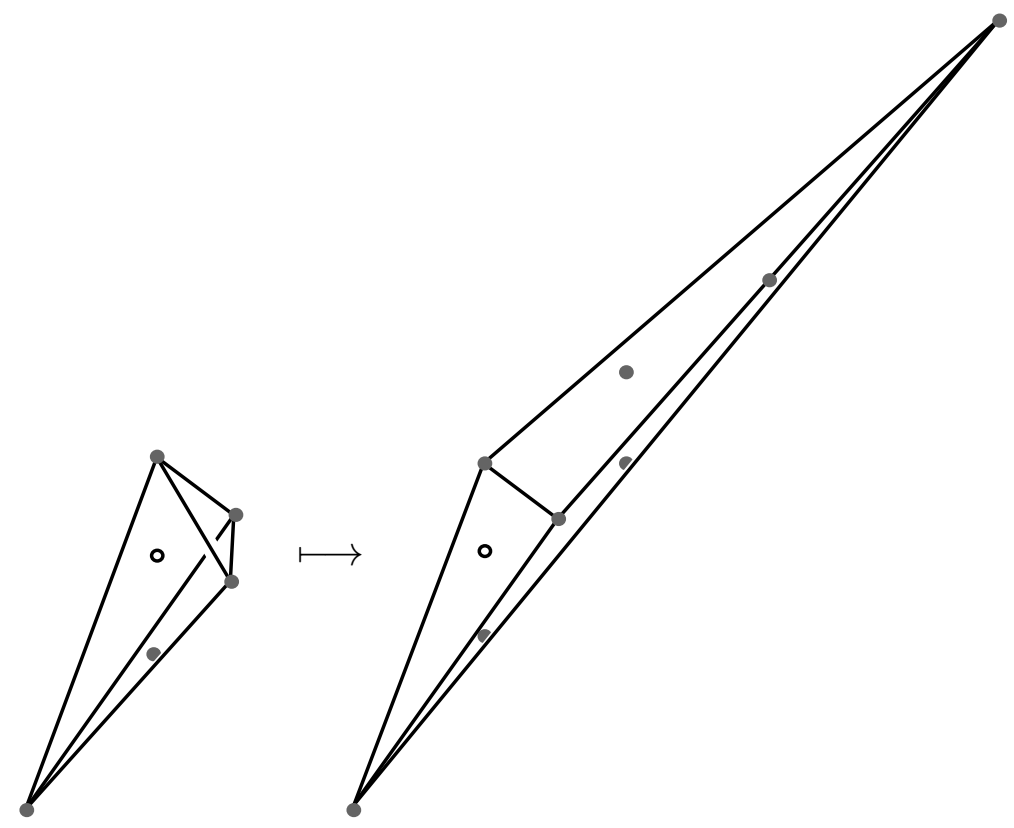

Figure 1: An edge mutation from the polytope corresponding to $\mathbb{P}(1,1,1,3)$, depicted on the left, to the polytope corresponding to $\mathbb{P}(1,1,4,6)$. This mutation is described in Example 6.

Proof. We first consider $w_{h_{\max }}(P)$. Suppose for a contradiction that $w_{h_{\max }}(P)$ is not a vertex of $P$. Then in particular it contains an edge $E_{1}$ of $P$. Let us pick an edge $E_{2}$ contained in $w_{h_{\min }}(P)$ (if such an edge does not exist then $w_{h_{\min }}(P)$ is a vertex and the mutation is trivial). Note that $E_{1}$ and $E_{2}$ cannot be parallel: if they were then the four endpoints of $E_{1}$ and $E_{2}$ would lie in a common two-dimensional affine subspace and thus would be affinely dependent; this contradicts the fact that $P$ is a simplex. It is an immediate consequence of the definition of mutation that $w_{h_{\max }}(Q)=w_{h_{\max }}(P)+h_{\max } F$. Recall that $F$ is a Minkowski factor of $w_{h_{\min }}(P)$. Because $P$ is a simplex, $w_{h_{\min }}(P)$ is also a simplex and so by [30, Result 13] $F$ is a dilation and translation of $w_{h_{\min }}(P)$. It follows that $h_{\max } F$ is also a dilation and translation of $w_{h_{\min }}(P)$. Let $E_{2}^{\prime}$ denote the edge of $h_{\max } F$ corresponding to the edge $E_{2}$ of $w_{h_{\min }}(P)$. Then $E_{1}$ and $E_{2}^{\prime}$ are not parallel, because $E_{1}$ and $E_{2}$ are not parallel. Thus the face $E_{1}+E_{2}^{\prime}$ of $w_{h_{\max }}(Q)=w_{h_{\max }}(P)+h_{\max } F$ is a quadrilateral. Since $Q$ is by assumption a simplex, and therefore all faces of $Q$ are simplices, this gives a contradiction. We conclude that $w_{h_{\max }}(P)$ consists of a single vertex.

Now consider the factor $F$. By the definition of factor there exists a lattice polytope $G_{h_{\min }}$ such that

$$
w_{h_{\text {min }}}(P)=G_{h_{\min }}+\left|h_{\min }\right| F .
$$

We claim that $G_{h_{\min }}$ is a point. After mutation we have $w_{h_{\min }}(Q)=G_{h_{\min }}$. As discussed above, we can mutate $Q$ back to $P$ by taking the height function $-w$ and the factor $F$. But $(-w)_{-h_{\min }}(Q)=w_{h_{\min }}(Q)=G_{h_{\min }}$ and we conclude from the first part of the proposition that $G_{h_{\min }}$ is a point: $G_{h_{\min }}=\{v\}$. It follows that $\left|h_{\min }\right| F=w_{h_{\min }}(P)-v$, 
and so $F$ is a translation of $\frac{1}{\left|h_{\min }\right|} w_{h_{\min }}(P)$ as required.

Definition 8. If $w_{h_{\min }}(P)$ has $k+1$ vertices then we say that the corresponding mutation is a mutation over a $k$-face. Cases of particular interest are $k=1$ and $k=n-1$, which we call mutations over edges and mutations over facets, respectively.

Lemma 9. Let $P$ be a Fano $n$-simplex, let $w \in M$ be a primitive height function, and let $F \subset N_{\mathbb{Q}}$ be a factor such that $Q:=\operatorname{mut}_{w}(P, F)$ is a simplex. Let $v \in \operatorname{vert}(P)$ be such that $w(v) \neq h_{\max }$ and $w(v) \neq h_{\min }$. Then $w(v)=0$.

Proof. Let us write $P=\operatorname{conv}\left\{v_{0}, \ldots, v_{n}\right\}$ and $w_{h_{\min }}(P)=\operatorname{conv}\left\{v_{1}, \ldots, v_{k}\right\}$. If $k=n$ then the statement holds vacuously, so let us assume that $k<n$. Without loss of generality we may assume that $\mathbf{0} \in \operatorname{vert}(F)$. In view of Lemma 7 we may assume further that $w_{h_{\max }}(P)=\left\{v_{0}\right\}$, that $w_{h_{\min }}(P)=v_{1}+\left|h_{\min }\right| F$, and that

$$
F:=\operatorname{conv}\left\{\mathbf{0}, \frac{1}{\left|h_{\min }\right|}\left(v_{2}-v_{1}\right), \ldots, \frac{1}{\left|h_{\min }\right|}\left(v_{k}-v_{1}\right)\right\} .
$$

Suppose for a contradiction that there exists some $v \in \operatorname{vert}(P)$ such that $w(v) \neq h_{\max }$, $w(v) \neq h_{\min }$, and $w(v) \neq 0$. Without loss of generality we can take $v=v_{n}$.

Suppose first that $w\left(v_{n}\right)>0$. Let $w^{\prime} \in M$ be a primitive lattice point such that $w^{\prime}\left(v_{i}\right)=0$ for $v_{i} \in\left\{v_{1}, \ldots, v_{n-1}\right\}$. We can choose $w^{\prime}$ so that $w^{\prime}\left(v_{0}\right)>0$ and $w^{\prime}\left(v_{n}\right)<0$. Let $h_{\max }^{\prime}=\sup \left\{w^{\prime}(p) \mid p \in P\right\}$ and $h_{\text {min }}^{\prime}=\inf \left\{w^{\prime}(p) \mid p \in P\right\}$. We see that $H_{w^{\prime}, h_{\max }^{\prime}} \cap P=$ $\left\{v_{0}\right\}$ and $H_{w^{\prime}, h_{\min }^{\prime}} \cap P=\left\{v_{n}\right\}$. Note that by the definition of $F, w^{\prime}(F)=0$ and so $w^{\prime}(x)=w^{\prime}(x+w(x) F)$ for all $x \in P($ with $w(x) \geqslant 0)$. Then $H_{w^{\prime}, h_{\max }^{\prime}} \cap Q=v_{0}+h_{\max } F=$ $v_{0}+w\left(v_{0}\right) F$ and $H_{w^{\prime}, h_{\min }^{\prime}} \cap Q=v_{n}+w\left(v_{n}\right) F$. Thus $v_{0}+w\left(v_{0}\right) F$ and $v_{n}+w\left(v_{n}\right) F$ are two faces of $Q$, with vertices $v_{0}+w\left(v_{0}\right) f$ and $v_{n}+w\left(v_{n}\right) f$ for $f \in \operatorname{vert}(F)$. Let $f_{1}, f_{2} \in \operatorname{vert}(F)$ be any two distinct vertices of $F$; these exist since otherwise $F$ is a point and the mutation is trivial. As $Q$ is a simplex

$$
F^{\prime}:=\operatorname{conv}\left\{v_{0}+w\left(v_{0}\right) f_{1}, v_{0}+w\left(v_{0}\right) f_{2}, v_{n}+w\left(v_{n}\right) f_{1}, v_{n}+w\left(v_{n}\right) f_{2}\right\}
$$

is a two-dimensional face of $Q$. But $F^{\prime}$ has four vertices and thus is not a simplex. This gives a contradiction.

Suppose instead that $w\left(v_{n}\right)<0$. By [1, Lemma 3.7] there exists a $v_{Q} \in \operatorname{vert}(Q)$ and $v_{F} \in \operatorname{vert}(F)$ such that $v_{n}=v_{Q}-w\left(v_{Q}\right) v_{F}$. Applying $w$ to this equation yields $w\left(v_{Q}\right)=w\left(v_{n}\right)<0$. We now have $\operatorname{mut}_{-w}(Q, F)=P$ but $-w\left(v_{Q}\right)>0$ which, by the preceding argument, gives a contradiction. It follows that $w\left(v_{n}\right)=0$.

Combining the previous two results gives a necessary and sufficient combinatorial condition for the existence of a mutation between simplices.

Lemma 10. Let $P$ be a Fano n-simplex and let $w \in M$ be a primitive height function. Let the vertices of $P$ be $\left\{v_{0}, v_{1}, \ldots, v_{n}\right\}$, ordered such that $w_{h_{\min }}(P)=\operatorname{conv}\left\{v_{1}, \ldots, v_{k}\right\}$. There exists a factor $F \subset N_{\mathbb{Q}}$ such that $Q:=\operatorname{mut}_{w}(P, F)$ is a simplex if and only if the following hold: 
1. $w_{h_{\max }}(P)$ is a vertex;

2. $h_{\min } \mid v_{i}-v_{1}$ for $i \in\{1, \ldots, k\}$;

3. if $v$ is a vertex of $P$ such that $w(v) \neq h_{\max }$ and $w(v) \neq h_{\min }$, then $w(v)=0$.

Proof. The "only if" direction is Lemmas 7 and 9. On the other hand, it is clear that if conditions (1)-(3) are satisfied, then defining

$$
F:=\operatorname{conv}\left\{\mathbf{0}, \frac{1}{\left|h_{\min }\right|}\left(v_{2}-v_{1}\right), \ldots, \frac{1}{\left|h_{\min }\right|}\left(v_{k}-v_{1}\right)\right\}
$$

gives a factor with respect to $w$. Let us label the vertices of $P=\operatorname{conv}\left\{v_{0}, \ldots, v_{n}\right\}$ so that:

$$
w\left(v_{0}\right)=h_{\max }, \quad w\left(v_{1}\right)=\ldots=w\left(v_{k}\right)=h_{\min }, \quad w\left(v_{k+1}\right)=\ldots=w\left(v_{n}\right)=0 .
$$

From $\left[1\right.$, Lemma 3.7] we have that $\operatorname{vert}(Q) \subseteq\left\{v_{0}^{\prime}, v_{1}^{\prime}, \ldots, v_{n}^{\prime}\right\}$, where:

$$
v_{i}^{\prime}= \begin{cases}v_{i} & \text { if } i=0, i=1, \text { or } i \in\{k+1, \ldots, n\} \\ v_{0}+\frac{h_{\max }}{\left|h_{\min }\right|}\left(v_{i}-v_{1}\right) & \text { if } i \in\{2, \ldots, k\} .\end{cases}
$$

Furthermore by [1, Proposition 3.11] we have that $Q$ is Fano, and hence is of maximal dimension in the $n$-dimensional lattice. It follows that $Q$ must have at least $n+1$ vertices. Thus $Q=\operatorname{conv}\left\{v_{0}^{\prime}, v_{1}^{\prime}, \ldots, v_{n}^{\prime}\right\}$, and so $Q$ is a simplex.

Example 11. Consider the 3-simplex

$$
P=\operatorname{conv}\{(1,-1,0),(-2,-2,-1),(-2,-2,1),(0,1,0)\}
$$

The weighted projective space associated to $P$ is $\mathbb{P}(1,1,4,8)$. Let $w=(1,0,0) \in M$. Lemma 10 implies that there is a factor $F$ such that $Q:=\operatorname{mut}_{w}(P, F)$ is the simplex:

$$
Q=\operatorname{conv}\{(1,-1,0),(-2,-2,-1),(1,-1,1),(0,1,0)\}
$$

The weighted projective space associated to $Q$ is $\mathbb{P}(1,1,1,4)$.

The main result of this paper is:

Theorem 12. Let $X$ and $Y$ be fake weighted projective spaces related by a mutation. Suppose that $P=\operatorname{conv}\left\{v_{0}, \ldots, v_{n}\right\} \subset N_{\mathbb{Q}}$ is the simplex corresponding to $X$, that $Q \subset N_{\mathbb{Q}}$ is the simplex corresponding to $Y$, and that the weights of $X$ are $\lambda_{0}, \lambda_{1}, \ldots, \lambda_{n}$. Let $w \in M$ be the primitive height function and $F \subset N_{\mathbb{Q}}$ be the factor such that $Q=\operatorname{mut}_{w}(P, F)$. Then we may relabel the vertices of $P$ such that $w_{h_{\max }}(P)=v_{0}, w_{h_{\min }}(P)=\operatorname{conv}\left\{v_{1}, \ldots, v_{k}\right\}$, and the weights of $Y$ are:

$$
\frac{1}{d}\left(\lambda_{0} \lambda_{1},\left(\lambda_{1}+\ldots+\lambda_{k}\right)^{2}, \lambda_{0} \lambda_{2}, \ldots, \lambda_{0} \lambda_{k}, \lambda_{k+1}\left(\lambda_{1}+\ldots+\lambda_{k}\right), \ldots, \lambda_{n}\left(\lambda_{1}+\ldots+\lambda_{k}\right)\right)
$$

where $d$ is a positive integer satisfying:

$$
d \cdot \frac{\operatorname{mult}(X)}{\operatorname{mult}(Y)}=\frac{\lambda_{0}^{k-1}}{\left(\lambda_{1}+\ldots+\lambda_{k}\right)^{k-2}}
$$


Proof. By Lemma 10 we have $Q=\operatorname{conv}\left\{v_{0}^{\prime}, v_{1}^{\prime}, \ldots, v_{n}^{\prime}\right\}$ where:

$$
v_{i}^{\prime}= \begin{cases}v_{i} & \text { if } i=0, i=1, \text { or } i \in\{k+1, \ldots, n\} \\ v_{0}+\frac{h_{\max }}{\left|h_{\min }\right|}\left(v_{i}-v_{1}\right) & \text { if } i \in\{2, \ldots, k\} .\end{cases}
$$

Since $P$ is Fano we may assume that the weights $\lambda_{0}, \lambda_{1}, \ldots, \lambda_{n}$ are well-formed. We normalise by setting $h:=\sum_{i=0}^{n} \lambda_{i}$ and $\lambda_{i}^{\prime}:=\frac{1}{h} \lambda_{i}$. Then $\sum_{i=0}^{n} \lambda_{i}^{\prime} v_{i}=\mathbf{0}, \sum_{i=0}^{n} \lambda_{i}^{\prime}=1$, and $\lambda_{i}^{\prime} \geqslant 0$ for all $i$. The sequence $\lambda_{0}^{\prime}, \lambda_{1}^{\prime}, \ldots, \lambda_{n}^{\prime}$ is unique with these properties; these are the normalised barycentric co-ordinates for $P$.

Since $Q$ is Fano there exist $\mu_{0}^{\prime}, \mu_{1}^{\prime}, \ldots, \mu_{n}^{\prime}$ such that $\sum_{i=0}^{n} \mu_{i}^{\prime} v_{i}^{\prime}=\mathbf{0}, \sum_{i=0}^{n} \mu_{i}^{\prime}=1$, and $\mu_{i}^{\prime} \geqslant 0$ for all $i$. From $\sum_{i=0}^{n} \mu_{i}^{\prime} v_{i}^{\prime}=\mathbf{0}$ and (1) we have that:

$$
\left(\mu_{0}^{\prime}+\sum_{i=2}^{k} \mu_{i}^{\prime}\right) v_{0}+\left(\mu_{1}^{\prime}-\frac{h_{\max }}{\left|h_{\min }\right|} \sum_{i=2}^{k} \mu_{i}^{\prime}\right) v_{1}+\sum_{i=2}^{k} \mu_{i}^{\prime} \frac{h_{\max }}{\left|h_{\min }\right|} v_{i}+\sum_{i=k+1}^{n} \mu_{i}^{\prime} v_{i}=\mathbf{0} .
$$

Let $\theta_{i}$ denote the coefficient of $v_{i}$ in the expression above. We claim that the $\theta_{i}$ are normalised barycentric co-ordinates. It is clear that $\sum_{i=0}^{n} \theta_{i}=\sum_{i=0}^{n} \mu_{i}^{\prime}=1$, and that $\theta_{0}, \theta_{2}, \theta_{3}, \ldots, \theta_{n} \geqslant 0$. It remains to check that $\theta_{1} \geqslant 0$. Suppose for a contradiction that $\theta_{1}<0$. Then we have:

$$
-\theta_{1} v_{1}=\theta_{0} v_{0}+\theta_{2} v_{2}+\ldots+\theta_{n} v_{n} \in \operatorname{cone}\left\{v_{0}, v_{2}, \ldots, v_{n}\right\}
$$

and $-\theta_{1}>0$, so a point on the ray from $\mathbf{0}$ through $v_{1}$ lies in the cone over $v_{0}, v_{2}, \ldots, v_{n}$. This contradicts the fact that $\mathbf{0}$ lies in the strict interior of $P$.

Hence the $\theta_{i}$ are normalised barycentric co-ordinates for $P$, and so by uniqueness we have $\theta_{i}=\lambda_{i}^{\prime}$ for all $i$. Solving these equations for $\mu_{i}^{\prime}$ yields:

$$
\mu_{i}^{\prime}= \begin{cases}\lambda_{0}^{\prime}-\frac{\left|h_{\min }\right|}{h_{\max }} \sum_{i=2}^{k} \lambda_{i}^{\prime} & \text { if } i=0 \\ \lambda_{1}^{\prime}+\sum_{i=2}^{k} \lambda_{i}^{\prime} & \text { if } i=1 \\ \frac{\left|h_{\min }\right|}{h_{\max }} \lambda_{i}^{\prime} & \text { if } i \in\{2, \ldots, k\} \\ \lambda_{i}^{\prime} & \text { if } i \in\{k+1, \ldots, n\} .\end{cases}
$$

Applying $w$ to both sides of the equation $\sum_{i=1}^{n} \lambda_{i} v_{i}=\mathbf{0}$, we find that

$$
\frac{\left|h_{\min }\right|}{h_{\max }}=\frac{\lambda_{0}}{\lambda_{1}+\ldots+\lambda_{k}}
$$

and thus:

$$
\mu_{i}^{\prime}= \begin{cases}\lambda_{0}^{\prime}-\frac{\lambda_{0}}{\lambda_{1}+\ldots+\lambda_{k}} \sum_{i=2}^{k} \lambda_{i}^{\prime} & \text { if } i=0 \\ \lambda_{1}^{\prime}+\sum_{i=2}^{k} \lambda_{i}^{\prime} & \text { if } i=1 \\ \frac{\lambda_{0}}{\lambda_{1}+\ldots+\lambda_{k}} \lambda_{i}^{\prime} & \text { if } i \in\{2, \ldots, k\} \\ \lambda_{i}^{\prime} & \text { if } i \in\{k+1, \ldots, n\}\end{cases}
$$


Now we can form integer weights by defining $\mu_{i}=h\left(\lambda_{1}+\ldots+\lambda_{k}\right) \mu_{i}^{\prime}$; this gives:

$$
\mu_{i}= \begin{cases}\lambda_{0} \lambda_{1} & \text { if } i=0 \\ \left(\lambda_{1}+\ldots+\lambda_{k}\right)^{2} & \text { if } i=1 \\ \lambda_{0} \lambda_{i} & \text { if } i \in\{2, \ldots, k\} \\ \lambda_{i}\left(\lambda_{1}+\ldots+\lambda_{k}\right) & \text { if } i \in\{k+1, \ldots, n\}\end{cases}
$$

These weights are integers, but they may not be well-formed. However since $Q$ is Fano, we know that the weights are well-formed if and only if they are reduced. Therefore it remains to divide through by their greatest common divisor, which we denote by $d$. Thus the weights of $Q$ are:

$$
\frac{1}{d}\left(\lambda_{0} \lambda_{1},\left(\lambda_{1}+\ldots+\lambda_{k}\right)^{2}, \lambda_{0} \lambda_{2}, \ldots, \lambda_{0} \lambda_{k}, \lambda_{k+1}\left(\lambda_{1}+\ldots+\lambda_{k}\right), \ldots, \lambda_{n}\left(\lambda_{1}+\ldots+\lambda_{k}\right)\right) .
$$

Consider now the degrees of $X$ and $Y$ :

$$
\begin{aligned}
\left(-K_{X}\right)^{n} & =\frac{\left(\lambda_{0}+\ldots+\lambda_{n}\right)^{n}}{\lambda_{0} \ldots \lambda_{n} \operatorname{mult}(X)} \\
\left(-K_{Y}\right)^{n} & =\frac{\frac{1}{d^{n}}\left(\lambda_{0} \lambda_{1}+\ldots+\lambda_{n}\left(\lambda_{1}+\ldots+\lambda_{k}\right)\right)^{n}}{\frac{1}{d^{n+1}}\left(\left(\lambda_{0} \lambda_{1}\right) \ldots \lambda_{n}\left(\lambda_{1}+\ldots+\lambda_{k}\right)\right) \operatorname{mult}(Y)}
\end{aligned}
$$

Since degree is preserved by mutation, we conclude that

$$
d \cdot \frac{\operatorname{mult}(X)}{\operatorname{mult}(Y)}=\frac{\lambda_{0}^{k-1}}{\left(\lambda_{1}+\ldots+\lambda_{k}\right)^{k-2}}
$$

as claimed.

Remark 13. When $\operatorname{mult}(X)=\operatorname{mult}(Y)$, and in particular if $X$ and $Y$ are weighted projective spaces, we have:

$$
d=\frac{\lambda_{0}^{k-1}}{\left(\lambda_{1}+\ldots+\lambda_{k}\right)^{k-2}}
$$

This gives an explicit expression for the weights after mutation in terms of the weights before mutation.

Remark 14. In the case of a mutation over a facet we see that the new weights are:

$$
\frac{1}{d}\left(\lambda_{0} \lambda_{1},\left(\lambda_{1}+\ldots+\lambda_{k}\right)^{2}, \lambda_{0} \lambda_{2}, \ldots, \lambda_{0} \lambda_{n}\right)
$$

Note that $\lambda_{0}$ divides $d$, because $\lambda_{0} \mid \lambda_{0} \lambda_{i}$ for $i=1, \ldots, n$ and the weights are well-formed. On the other hand, after dividing through by $\lambda_{0}$ we obtain well-formed weights, and so in fact $d=\lambda_{0}$. In this case, therefore, we obtain an explicit formula for how the multiplicity changes:

$$
\frac{\operatorname{mult}(X)}{\operatorname{mult}(Y)}=\left(\frac{\lambda_{0}}{\lambda_{1}+\ldots+\lambda_{n}}\right)^{n-2}
$$




\section{Multiplicity-preserving Mutations}

The following result places a strong restriction on which mutations of fake weighted projective spaces can preserve multiplicity.

Theorem 15. Any non-trivial multiplicity-preserving mutation between fake weighted projective spaces $X$ and $Y$ is a mutation over an edge.

Proof. Let $P=\operatorname{conv}\left\{v_{0}, v_{1}, \ldots, v_{n}\right\}$ be the simplex associated to $X$, and let $\lambda_{0}, \lambda_{1}, \ldots, \lambda_{n}$ be the corresponding weights. Let $Q=\operatorname{conv}\left\{v_{0}^{\prime}, v_{1}^{\prime}, \ldots, v_{n}^{\prime}\right\}$ be the simplex associated to $Y$, and let $\lambda_{0}^{\prime}, \lambda_{1}^{\prime}, \ldots, \lambda_{n}^{\prime}$ be the corresponding weights. Suppose for a contradiction that $P$ and $Q$ are related by a non-trivial mutation over a $k$-face, for some $k>2$. By Remark 13, after reordering weights if necessary, we have:

$$
\begin{aligned}
& \left(\mu_{0}, \ldots, \mu_{n}\right)= \\
& \quad \frac{1}{d}\left(\left(\lambda_{1}+\ldots+\lambda_{k}\right)^{2}, \lambda_{0} \lambda_{1}, \lambda_{0} \lambda_{2}, \ldots, \lambda_{0} \lambda_{k}, \lambda_{k+1}\left(\lambda_{1}+\ldots+\lambda_{k}\right), \ldots, \lambda_{n}\left(\lambda_{1}+\ldots+\lambda_{k}\right)\right)
\end{aligned}
$$

where:

$$
d=\frac{\lambda_{0}^{k-1}}{\left(\lambda_{1}+\ldots+\lambda_{k}\right)^{k-2}}
$$

We recall from (2) that

$$
\frac{h_{\max }}{\left|h_{\min }\right|}=\frac{\lambda_{1}+\ldots+\lambda_{k}}{\lambda_{0}}
$$

and write $h_{\max } /\left|h_{\min }\right|=A / B$ with $A$ and $B$ coprime integers. So, for $i \in\{1, \ldots, k\}$, we have:

$$
\mu_{i}=\frac{\lambda_{0} \lambda_{i}}{d}=\lambda_{i}\left(\frac{\lambda_{1}+\ldots+\lambda_{k}}{\lambda_{0}}\right)^{k-2}=\lambda_{i}\left(\frac{A}{B}\right)^{k-2}
$$

Since $A$ and $B$ are coprime and $k>2$, we have that $B \mid \lambda_{i}$. Similarly for $i \in\{k+1, \ldots, n\}$ we have:

$$
\mu_{i}=\frac{\lambda_{i}\left(\lambda_{1}+\ldots+\lambda_{k}\right)}{d}=\lambda_{i}\left(\frac{\lambda_{1}+\ldots+\lambda_{k}}{\lambda_{0}}\right)^{k-1}=\lambda_{i}\left(\frac{A}{B}\right)^{k-1}
$$

and so in this case too $B \mid \lambda_{i}$. However $\lambda_{1}, \lambda_{2}, \ldots, \lambda_{n}$ are coprime, because the weights of $X$ are well-formed, and therefore $B=1$.

Since $h_{\max } /\left|h_{\min }\right|=A / B=A$ is an integer, we have that $\lambda_{0} \mid \lambda_{1}+\ldots+\lambda_{k}$. It follows that $\lambda_{0} \mid d \mu_{i}$ for all $i$, and since the weights $\mu_{i}$ are reduced we conclude that $\lambda_{0} \mid d$. Then

$$
\frac{d}{\lambda_{0}}=\left(\frac{\lambda_{0}}{\lambda_{1}+\ldots+\lambda_{k}}\right)^{k-2}
$$

is an integer. Taking the $(k-2)$ th root (recall that $k>2$ ) we see that $\frac{\lambda_{0}}{\lambda_{1}+\ldots+\lambda_{k}}$ is an integer, and hence that $\lambda_{1}+\ldots+\lambda_{k} \mid \lambda_{0}$. Thus $\lambda_{1}+\ldots+\lambda_{k}=\lambda_{0}$. Substituting this into our expression for the $\mu_{i}$ shows that the mutation is trivial, which is a contradiction. 
Corollary 16. Suppose that $X$ is a weighted projective space that admits a non-trivial mutation to another weighted projective space. Let $\lambda_{0}$ be the weight corresponding to the vertex $w_{h_{\max }}(P)$, and let $\lambda_{1}, \lambda_{2}$ be the weights corresponding to the vertices of the edge $w_{h_{\min }}(P)$. Then:

$$
\begin{aligned}
& \text { 1. } \lambda_{0} \mid\left(\lambda_{1}+\lambda_{2}\right)^{2} \\
& \text { 2. } \operatorname{gcd}\left\{\lambda_{1}, \lambda_{2}\right\} \mid \lambda_{0}
\end{aligned}
$$

Proof. The mutation is an edge mutation, and so Theorem 12 implies both that $d=\lambda_{0}$ and that $d \mid\left(\lambda_{1}+\lambda_{2}\right)^{2}$. This proves (1). Looking again at Theorem 12 and using wellformedness of weights, we see that $\operatorname{gcd}\left\{\lambda_{1}, \lambda_{2}\right\} \mid d$. This proves (2).

Remark 17. Let $X$ be a fake weighted projective plane. Akhtar and Kasprzyk characterise mutations from $X$ to other fake weighted projective planes in terms of solutions to an associated Diophantine equation [2, Proposition 3.12]. Their argument relies on the fact that, for lattice triangles, the square-free parts of the weights are preserved (up to reordering) under mutation. This phenomenon does not persist in higher dimensions:

1. Example 6 above shows that, in general, neither the square-free parts of the weights nor the $k$ th-power-free parts of the weights nor the square parts of the weights nor the $k$ th-power parts of the weights are preserved.

2. Example 11 above shows that, in general, neither the $n$ th-power-free parts nor the $n$ th-power parts of the weights are preserved.

\section{Canonical and Terminal Singularities}

Terminal and canonical singularities were introduced by Miles Reid; they play a fundamental role in birational geometry $[27,28,29]$. Terminal singularities form the smallest class of singularities that must be allowed if one wishes to construct minimal models in dimensions three or more. Canonical singularities can be regarded as the limit of terminal singularities; they arise naturally as the singularities occurring on canonical models of varieties of general type. From the toric viewpoint, terminal and canonical singularities have a particularly elegant combinatorial description. A toric singularity corresponds to a strictly convex rational polyhedral cone $\sigma \subset N_{\mathbb{Q}}$ [13]. The cone $\sigma$ is terminal if and only if:

1. the lattice points $\rho_{1}, \ldots, \rho_{m}$ corresponding to the primitive generators of the rays of $\sigma$ are contained in an affine hyperplane $H_{u}:=\left\{v \in N_{\mathbb{Q}} \mid u(v)=1\right\}$ for some $u \in M_{\mathbb{Q}}$

2. with the exception of the origin $\mathbf{0}$ and the generators $\rho_{i}$ of the rays, no other lattice points of $N$ are contained in the part of $\sigma$ on or under $H_{u}$, i.e.

$$
N \cap \sigma \cap\left\{v \in N_{\mathbb{Q}} \mid u(v) \leqslant 1\right\}=\left\{\mathbf{0}, \rho_{1}, \ldots, \rho_{m}\right\}
$$


The cone $\sigma$ is canonical if and only if (1) holds and

(ii') the origin $\mathbf{0}$ is the only lattice point contained in the part of $\sigma$ under $H_{u}$, i.e.

$$
N \cap \sigma \cap\left\{v \in N_{\mathbb{Q}} \mid u(v)<1\right\}=\{\mathbf{0}\}
$$

When the hyperplane $H_{u}$ in condition (1) corresponds to a lattice point $u \in M$, the singularity is called Gorenstein.

\section{Weighted Projective Spaces With Canonical Sin- gularities}

We now apply our results to the study of weighted projective spaces with canonical singularities, giving some geometric context, in terms of mutations, for a recent combinatorial result that characterises the fake weighted projective spaces of maximal degree with at worst canonical singularities [4]. We say that a (fake) weighted projective space with at worst canonical singularities is a canonical (fake) weighted projective space.

Remark 18. If $\mathbb{P}\left(\lambda_{0}, \ldots, \lambda_{n}\right) / G$ is a canonical fake weighted projective space then the weighted projective space $\mathbb{P}\left(\lambda_{0}, \ldots, \lambda_{n}\right)$ has canonical singularities. Thus a canonical fake weighted projective space of maximal degree is necessarily a weighted projective space.

Definition 19. The Sylvester numbers $y_{0}, y_{1}, y_{2}, \ldots$ are defined by:

$$
y_{n}= \begin{cases}2 & \text { if } n=0 \\ 1+\prod_{i=0}^{n-1} y_{i} & \text { otherwise }\end{cases}
$$

We set $t_{n}:=y_{n}-1$.

Lemma 20 ([31]).

1. The Sylvester numbers are pairwise coprime;

2. If $n \geqslant 1$ and $i<n$ then $t_{n} / y_{i}$ is an integer. Furthermore if $p$ is a prime dividing $y_{i}$ then $p \mid t_{n} / y_{j}$ for $j<n, j \neq i$, and $p \nmid t_{n} / y_{i}$;

3. We have:

$$
\sum_{i=0}^{n-1} \frac{1}{y_{i}}=\frac{t_{n}-1}{t_{n}}
$$

Define:

$$
X_{n}:=\mathbb{P}\left(1,1, \frac{2 t_{n-1}}{y_{n-2}}, \ldots, \frac{2 t_{n-1}}{y_{0}}\right)
$$

$X_{n}$ is an $n$-dimensional canonical weighted projective space. Averkov, Krümpelmann, and Nill [4] have proved that, if $n \geqslant 4$, then $X_{n}$ is the unique $n$-dimensional canonical weighted projective space of maximum degree; this generalizes the corresponding result 
for Gorenstein weighted projective spaces, which is due to Nill [25]. In dimension three there are precisely two canonical weighted projective spaces of maximum degree, and these are connected by a mutation: see Example 6 . We next determine all weighted projective spaces $X_{n}^{(m, a)}$ that might be connected to $X_{n}$ by a sequence of mutations through weighted projective spaces, and show that none of the $X_{n}^{(m, a)}$ are canonical.

Proposition 21. Define integers $\lambda_{i}^{(m, a)}$, where $0 \leqslant i \leqslant n$ and $0 \leqslant a \leqslant n-2$, by:

$$
\lambda_{i}^{(0, a)}= \begin{cases}1 & \text { if } i=0 \\ \frac{2 t_{n-1}}{y_{a}} & \text { if } i=1 \\ 1 & \text { if } i=2 \\ \frac{2 t_{n-1}}{y_{k_{i}}} & \text { otherwise }\end{cases}
$$

where $\left\{k_{3}, \ldots, k_{n}\right\}$ is $\{0,1, \ldots, \widehat{a}, \ldots, n-2\}$ with a omitted, and:

$$
\lambda_{i}^{(m, a)}= \begin{cases}\lambda_{2}^{(m-1, a)} & \text { if } i=0 \\ \lambda_{1}^{(m-1, a)} & \text { if } i=1 \\ \frac{\left(\lambda_{1}^{(m-1, a)}+\lambda_{2}^{(m-1, a)}\right)^{2}}{\lambda_{0}^{(m-1, a)}} & \text { if } i=2 \\ \frac{\lambda_{i}^{(m-1, a)}\left(\lambda_{1}^{(m-1, a)}+\lambda_{2}^{(m-1, a)}\right)}{\lambda_{0}^{(m-1, a)}} & \text { otherwise }\end{cases}
$$

for $m \geqslant 1$. Set:

$$
X_{n}^{(m, a)}:=\mathbb{P}\left(\lambda_{0}^{(m, a)}, \lambda_{1}^{(m, a)}, \ldots, \lambda_{n}^{(m, a)}\right)
$$

Then:

1. $X_{n}^{(0, a)}=X_{n}$ for all $a$;

2. if $m>0$ and if $X$ is a weighted projective space with a non-trivial mutation to $X_{n}^{(m, a)}$ then either $X=X_{n}^{(m-1, a)}$ or $X=X_{n}^{(m+1, a)}$;

3. $X_{n}^{(m, a)}$ is not canonical for any $m \geqslant 1$.

In other words, the graph of mutations between weighted projective spaces starting at $X_{n}$ is a subtree of the graph depicted in Figure 2, and the only canonical weighted projective space in this graph is $X_{n}$.

Proof. Statement (1) is trivial. For $(3)$, recall that $\mathbb{P}\left(\mu_{0}, \ldots, \mu_{n}\right)$ is canonical if and only if

$$
\sum_{i=0}^{n}\left\{\frac{\mu_{i} \kappa}{h}\right\} \in\{1, \ldots, n-1\}
$$

for every $\kappa \in\{2, \ldots, h-2\}$, where $\{x\}$ denotes the fractional part of $x$ and $h$ is the sum of the $\mu_{i}$ [23, Proposition 2.5]. This fails for $m \geqslant 1$ and $\kappa=h^{(m, a)}-\lambda_{1}^{(m, a)}-\lambda_{2}^{(m-1, a)}$, where $h^{(m, a)}$ is the sum of the weights of $X_{n}^{(m, a)}$ : see Lemma 37 . 


\begin{tabular}{cccc}
\multicolumn{4}{c}{$X_{n}$} \\
\hline$X_{n}^{(1,0)}$ & $X_{n}^{(1,1)}$ & $\ldots$ & $X_{n}^{(1, n-2)}$ \\
$\mid$ & $\mid$ & $\mid$ & $\mid$ \\
$X_{n}^{(2,0)}$ & $X_{n}^{(2,1)}$ & $\ldots$ & $X_{n}^{(2, n-2)}$ \\
$\mid$ & $\mid$ & $\mid$ & $\mid$ \\
$X_{n}^{(3,0)}$ & $X_{n}^{(3,1)}$ & $\ldots$ & $X_{n}^{(3, n-2)}$ \\
$\mid$ & $\mid$ & $\mid$ & $\vdots$ \\
$\vdots$ & $\vdots$ & $\vdots$ & $\vdots$
\end{tabular}

Figure 2: The graph of mutations as described in Proposition 21.

It remains to prove (2). Suppose first that there is a non-trivial mutation from $X_{n}$ to a weighted projective space $X$. By Theorem 15, this must be an edge mutation. Let $P$ be the simplex associated to $X_{n}$, let $\mu_{0}$ denote the weight associated to the vertex $w_{h_{\max }}(P)$, and let $\mu_{1}, \mu_{2}$ be the weights associated to the vertices of $w_{h_{\min }}(P)$. We consider the possible values of $\mu_{0}, \mu_{1}, \mu_{2}$.

Case 1. $\mu_{0} \neq 1$. Then $\mu_{0}=2 t_{n-1} / y_{a_{0}}$ for some $a_{0} \in\{0, \ldots, n-2\}$.

Case 1.1. $\mu_{1}=1, \mu_{2}=1$. Since $n \geqslant 4$, there exist $a_{1}, a_{2} \in\{0,1, \ldots, n-2\}$ distinct such that $y_{a_{1}} \neq y_{a_{0}}$ and $y_{a_{2}} \neq y_{a_{0}}$. At least one of $y_{a_{1}}$ or $y_{a_{2}}$ is not equal to 2 , and hence is a divisor of $\mu_{0}$ not equal to 2 or 4 . But $\left(\mu_{1}+\mu_{2}\right)^{2}=4$, and this contradicts Corollary 16(1). Case 1.2. $\mu_{1} \neq 1, \mu_{2}=1$. Then $\mu_{1}=2 t_{n-1} / y_{a_{1}}$ for some $a_{1} \neq a_{0}$. Choose $a_{2}$ not equal to $a_{0}$ or $a_{1}$, and let $p$ be a prime dividing $y_{a_{2}}$. Lemma 20(2) imples that $p$ divides both $2 t_{n-1} / y_{a_{0}}$ and $2 t_{n-1} / y_{a_{1}}$. So $p$ does not divide $\left(2 t_{n-1} / y_{a_{1}}+1\right)^{2}$, and this contradicts Corollary 16(1).

Case 1.3. $\mu_{1} \neq 1, \mu_{2} \neq 1$. Then $\mu_{1}=2 t_{n-1} / y_{a_{1}}$ and $\mu_{2}=2 t_{n-1} / y_{a_{2}}$ for $a_{1}, a_{2}$ distinct and not equal to $a_{0}$. Suppose first that $y_{a_{0}} \neq 2$, and let $p$ be a prime dividing $y_{a_{0}}$. Lemma 20(2) implies that $p \mid \operatorname{gcd}\left\{\mu_{1}, \mu_{2}\right\}$ and $p \nmid \mu_{0}$, contradicting Corollary 16(2). On the other hand if $y_{a_{0}}=2$ then the same argument with $p=2$ shows that $4 \mid \operatorname{gcd}\left\{\mu_{1}, \mu_{2}\right\}$ and $4 \backslash \mu_{0}$, again contradicting Corollary 16(2)

Case 2. $\mu_{0}=1$.

Case 2.1. $\mu_{1} \neq 1, \mu_{2}=1$. Then $\mu_{1}=2 t_{n-1} / y_{a}$ for some $a \in\{0, \ldots, n-2\}$, and Theorem 12 implies that $X=X_{n}^{(1, a)}$.

Case 2.2. $\mu_{1} \neq 1, \mu_{2} \neq 1$. Then $\mu_{1}=2 t_{n-1} / y_{a_{1}}, \mu_{2}=2 t_{n-1} / y_{a_{2}}$ for $a_{1}, a_{2}$ distinct. Choose $a_{0}$ not equal to $a_{1}$ or $a_{2}$, and let $p$ be a prime dividing $y_{a_{0}}$. Lemma 20(2) implies that $p \mid \operatorname{gcd}\left\{\mu_{1}, \mu_{2}\right\}$ and $p \nmid \mu_{0}$, contradicting Corollary 16(2).

This completes the proof in the case where $m=0$.

Suppose now that $m>1$, and that there is a non-trivial mutation from $X_{n}^{(m, a)}$ to a weighted projective space $X$. Theorem 15 implies that this is an edge mutation. Let $P$ be the simplex corresponding to $X_{n}^{(m, a)}$, let $\mu_{0}$ denote the weight associated to the vertex $w_{h_{\max }}(P)$, and let $\mu_{1}, \mu_{2}$ denote the weights associated to the vertices of $w_{h_{\min }}(P)$. To 
declutter the notation, write $\lambda_{i}$ for the weight $\lambda_{i}^{(m, a)}$ of $X_{n}^{(m, a)}$. We consider the possible values of $\mu_{0}, \mu_{1}, \mu_{2}$ in turn.

Example Case. $\mu_{0}=\lambda_{i}$ with $i \geqslant 3, \mu_{1}=\lambda_{1}, \mu_{2}=\lambda_{2}$. By Lemma 26 we have that $\mu_{0}$ and $\mu_{2}$ share a common factor that does not divide $\mu_{1}$, and so does not divide $\mu_{1}+\mu_{2}$. Thus $\mu_{0} \backslash\left(\mu_{1}+\mu_{2}\right)$, contradicting Corollary 16(1).

The remaining cases are entirely analogous. We arrive at a contradiction in all but two cases, as summarized in Table 1 below; here $\lambda_{i}, \lambda_{j}, \lambda_{k}$ denote distinct elements of $\{3, \ldots, n\}$. The case $\mu_{0}=\lambda_{0}, \mu_{1}=\lambda_{1}, \mu_{2}=\lambda_{2}$ yields $X_{n}^{(m+1, a)}$, and the case $\mu_{0}=$ $\lambda_{2}, \mu_{1}=\lambda_{0}, \mu_{2}=\lambda_{1}$ yields $X_{n}^{(m-1, a)}$. This completes the proof in the case where $m>1$.

\begin{tabular}{|c|c|c|c|c|c|c|c|c|c|}
\hline$\mu_{0}$ & $\mu_{1}$ & $\mu_{2}$ & Contradicts & Using & $\mu_{0}$ & $\mu_{1}$ & $\mu_{2}$ & Contradicts & Using \\
\hline$\lambda_{0}$ & $\lambda_{1}$ & $\lambda_{2}$ & - & - & $\lambda_{2}$ & $\lambda_{1}$ & $\lambda_{i}$ & Corollary 16(1) & Lemma 27 \\
\hline$\lambda_{0}$ & $\lambda_{1}$ & $\lambda_{i}$ & Corollary 16(1) & Lemma 28 & $\lambda_{2}$ & $\lambda_{i}$ & $\lambda_{j}$ & Corollary 16(2) & Lemma 29 \\
\hline$\lambda_{0}$ & $\lambda_{2}$ & $\lambda_{i}$ & Corollary 16(1) & Lemma & $\lambda_{i}$ & $\lambda_{0}$ & $\lambda_{1}$ & Corollary 16(1) & Lemma 27 \\
\hline$\lambda_{0}$ & $\lambda_{i}$ & $\lambda_{j}$ & Corollary $16(2)$ & Lemma & $\lambda_{i}$ & $\lambda_{0}$ & $\lambda_{2}$ & Corollary 16(1) & Lemma 27 \\
\hline$\lambda_{1}$ & $\lambda_{0}$ & $\lambda_{2}$ & Corollary 16(1) & Lemma 31 & $\lambda_{i}$ & $\lambda_{0}$ & $\lambda_{j}$ & Corollary 16(1) & Lemma 27 \\
\hline$\lambda_{1}$ & $\lambda_{0}$ & $\lambda_{i}$ & Corollary 16(1) & Lemma 30 & $\lambda_{i}$ & $\lambda_{1}$ & $\lambda_{2}$ & Corollary 16(1) & Lemma 27 \\
\hline$\lambda_{1}$ & $\lambda_{2}$ & $\lambda_{i}$ & Corollary 16(1) & Lemma 30 & $\lambda_{i}$ & $\lambda_{1}$ & $\lambda_{j}$ & Corollary 16(1) & Lemma 27 \\
\hline$\lambda_{1}$ & $\lambda_{i}$ & $\lambda_{j}$ & Corollary $16(1)$ & Lemma 30 & $\lambda_{i}$ & $\lambda_{2}$ & $\lambda_{j}$ & Corollary 16(1) & Lemma 28 \\
\hline$\lambda_{2}$ & $\lambda_{0}$ & $\lambda_{1}$ & - & - & $\lambda_{i}$ & $\lambda_{j}$ & $\lambda_{k}$ & Corollary $16(1)$ & Lemma 30 \\
\hline$\lambda_{2}$ & $\lambda_{0}$ & $\lambda_{i}$ & Corollary 16(1) & Lemma 27 & & & & & \\
\hline
\end{tabular}

Table 1: A summary of the argument in the case where $m>1$.

Suppose now that $m=1$. We can argue exactly as for $m>1$, except in those cases where Lemma 28 is used. We consider these three cases separately. As before, write $\lambda_{i}$ for $\lambda_{i}^{(m, a)}$.

Case 3. $\mu_{0}=\lambda_{0}, \mu_{1}=\lambda_{1}, \mu_{2}=\lambda_{i}$ with $i \geqslant 3$. Then $\mu_{0}=1, \mu_{1}=2 t_{n-1} / y_{a}$, and $\mu_{2}=\frac{2 t_{n-1}}{y_{a_{i}}}\left(\frac{2 t_{n-1}}{y_{a}}+1\right)$. Choose $a_{j}$ not equal to $a$ or $a_{i}$, and let $p$ be a prime dividing $y_{a_{j}}$. Then $p$ divides $\mu_{1}$ and $\mu_{2}$ but does not divide $\mu_{0}$, contradicting Corollary 16(2).

Case 4. $\mu_{0}=\lambda_{0}, \mu_{1}=\lambda_{2}, \mu_{2}=\lambda_{i}$ with $i \geqslant 3$. Then $\mu_{0}=1, \mu_{1}=\left(2 t_{n-1} / y_{a}+1\right)^{2}$, and $\mu_{2}=\frac{2 t_{n-1}}{y_{a_{i}}}\left(\frac{2 t_{n-1}}{y_{a}}+1\right)$. Let $p$ be a prime dividing $2 t_{n-1} / y_{a}+1$. Then $p$ divides $\mu_{1}$ and $\mu_{2}$ but does not divide $\mu_{0}$, contradicting Corollary 16(2).

Case 5. $\mu_{0}=\lambda_{i}$ with $i \geqslant 3, \mu_{1}=\lambda_{2}, \mu_{2}=\lambda_{j}$ with $j \geqslant 3$. Then $\mu_{0}=\frac{2 t_{n-1}}{y_{a_{i}}}\left(\frac{2 t_{n-1}}{y_{a}}+1\right)$, $\mu_{1}=\left(2 t_{n-1} / y_{a}+1\right)^{2}$, and $\mu_{2}=\frac{2 t_{n-1}}{y_{a_{j}}}\left(\frac{2 t_{n-1}}{y_{a}}+1\right)$. Let $p$ be a prime dividing $y_{a}$. Then $p$ divides $\mu_{0}$ and $\mu_{2}$ (Lemma 20) but does not divide $\mu_{1}$ (Lemma 24). This contradicts Corollary 16(1).

This completes the proof in the case where $m=1$. 


\section{Weighted Projective Spaces With Terminal Singu- larities}

A (fake) weighted projective space with at worst terminal singularities is called a terminal (fake) weighted projective space. As before, a terminal fake weighted projective space of maximal degree is necessarily a weighted projective space. We now give a conjectural classification of terminal weighted projective spaces of maximal degree, and analyse them in terms of mutations.

In dimension 3 , the unique terminal weighted projective space of maximum degree is $\mathbb{P}^{3}$. In dimension 4, Kasprzyk has shown [23, Lemma 3.5] that the unique terminal weighted projective space of maximum degree is:

$$
\mathbb{P}(1,1,6,14,21)=\mathbb{P}\left(1,1, \frac{t_{3}}{y_{2}}, \frac{t_{3}}{y_{1}}, \frac{t_{3}}{y_{0}}\right)
$$

The classification problem for terminal weighted projective spaces of maximum degree in dimensions 5 and higher is open. The space

$$
X_{n}=\mathbb{P}\left(1,1, \frac{t_{n-1}}{y_{n-2}}, \ldots, \frac{t_{n-1}}{y_{0}}\right)
$$

is terminal [23, Lemma 3.7] and it seems reasonable to conjecture that, for $n \geqslant 4, X_{n}$ is the unique terminal weighted projective space of maximum degree. Note that the methods of [4] do not apply to this problem, as they are unable to distinguish between canonical and terminal singularities (they are insensitive to lattice points on the boundary of a simplex).

Proposition 22. Define integers $\lambda_{i}^{(m, a)}$, where $0 \leqslant i \leqslant n$ and $0 \leqslant a \leqslant n-2$, by:

$$
\lambda_{i}^{(0, a)}= \begin{cases}1 & \text { if } i=0 \\ \frac{t_{n-1}}{y_{a}} & \text { if } i=1 \\ 1 & \text { if } i=2 \\ \frac{t_{n-1}}{y_{k_{i}}} & \text { otherwise }\end{cases}
$$

where $\left\{k_{3}, \ldots, k_{n}\right\}$ is $\{0,1, \ldots, \widehat{a}, \ldots, n-2\}$ with a omitted, and:

$$
\lambda_{i}^{(m, a)}= \begin{cases}\lambda_{2}^{(m-1, a)} & \text { if } i=0 \\ \lambda_{1}^{(m-1, a)} & \text { if } i=1 \\ \frac{\left(\lambda_{1}^{(m-1, a)}+\lambda_{2}^{(m-1, a)}\right)^{2}}{\lambda_{0}^{(m-1, a)}} & \text { if } i=2 \\ \frac{\lambda_{i}^{(m-1, a)}\left(\lambda_{1}^{(m-1, a)}+\lambda_{2}^{(m-1, a)}\right)}{\lambda_{0}^{(m-1, a)}} & \text { otherwise }\end{cases}
$$

for $m \geqslant 1$. Suppose that $n \geqslant 5$. Set:

$$
X_{n}^{(m, a)}:=\mathbb{P}\left(\lambda_{0}^{(m, a)}, \lambda_{1}^{(m, a)}, \ldots, \lambda_{n}^{(m, a)}\right)
$$

Then: 
1. $X_{n}^{(0, a)}=X_{n}$ for all $a$;

2. if $m>0$ and if $X$ is a weighted projective space with a non-trivial mutation to $X_{n}^{(m, a)}$ then either $X=X_{n}^{(m-1, a)}$ or $X=X_{n}^{(m+1, a)}$;

3. $X_{n}^{(m, a)}$ is not terminal for any $m \geqslant 1$.

In other words, the graph of mutations between weighted projective spaces starting at $X_{n}$ is a subtree of the graph depicted in Figure 3, and the only terminal weighted projective space in this graph is $X_{n}$.

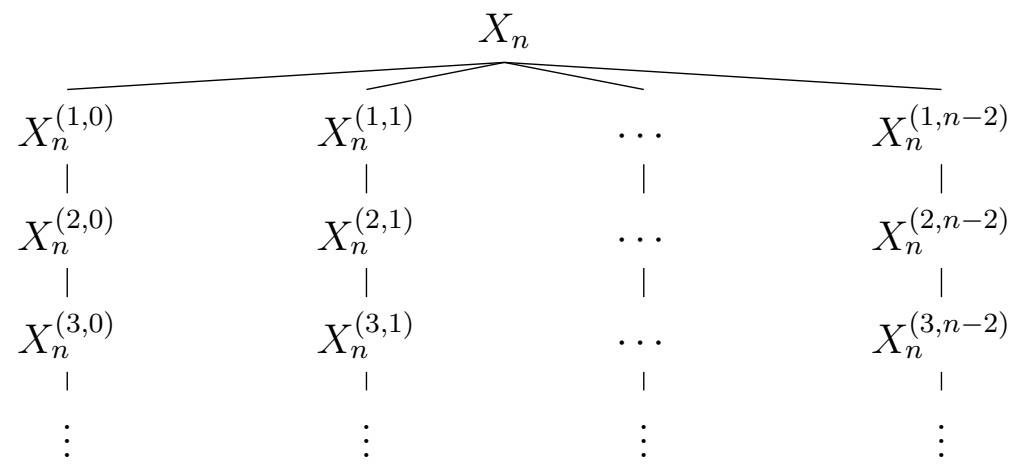

Figure 3: The graph of mutations as described in Proposition 22.

Proof. Statement (1) is trivial. For $(3)$, recall that $\mathbb{P}\left(\mu_{0}, \ldots, \mu_{n}\right)$ is terminal if and only if

$$
\sum_{i=0}^{n}\left\{\frac{\mu_{i} \kappa}{h}\right\} \in\{2, \ldots, n-1\}
$$

for every $\kappa \in\{2, \ldots, h-2\}$, where $\{x\}$ denotes the fractional part of $x$ and $h$ is the sum of the $\mu_{i}$ [23, Proposition 2.3]. This fails for $m \geqslant 1$ and $\kappa=h^{(m, a)}-h^{(m-1, a)}$, where $h^{(m, a)}$ is the sum of the weights of $X_{n}^{(m, a)}$ : see Lemma 39 .

It remains to prove (2). This is analogous to the proof of Proposition 21(2). The analogs of Lemmas 25-28 and Lemmas 30, 31 hold for these weights $\lambda_{i}^{(m, a)}$ too, with almost identical proofs, and Lemma 38 functions as a replacement for Lemma 29. Suppose first that $m>1$ and that there is a non-trivial mutation from $X_{n}^{(m, a)}$ to a weighted projective space $X$. By Theorem 15, this must be an edge mutation. Let $P$ be the simplex associated to $X_{n}^{(m, a)}$, let $\mu_{0}$ denote the weight associated to the vertex $w_{h_{\max }}(P)$, and let $\mu_{1}, \mu_{2}$ be the weights associated to the vertices of $w_{h_{\min }}(P)$. To declutter the notation, we again write $\lambda_{i}$ for the weight $\lambda_{i}^{(m, a)}$ of $X_{n}^{(m, a)}$. We consider the possible values of $\mu_{0}, \mu_{1}, \mu_{2}$ in turn, arriving at a contradiction in all but two cases. This is summarized in Table 2 below, where $\lambda_{i}, \lambda_{j}, \lambda_{k}$ denote distinct elements of $\{3, \ldots, n\}$. The case $\mu_{0}=\lambda_{0}, \mu_{1}=\lambda_{1}, \mu_{2}=\lambda_{2}$ yields $X_{n}^{(m+1, a)}$, and the case $\mu_{0}=\lambda_{2}, \mu_{1}=\lambda_{0}, \mu_{2}=\lambda_{1}$ yields $X_{n}^{(m-1, a)}$. 


\begin{tabular}{|c|c|c|c|c|c|c|c|c|c|}
\hline$\mu_{0}$ & $\mu_{1}$ & $\mu_{2}$ & Contradicts & Using & $\mu_{0}$ & $\mu_{1}$ & $\mu_{2}$ & Contradicts & Using \\
\hline$\lambda_{0}$ & $\lambda_{1}$ & $\lambda_{2}$ & - & - & $\lambda_{2}$ & $\lambda_{1}$ & $\lambda_{i}$ & Corollary $16(1)$ & Lemma 27 \\
\hline$\lambda_{0}$ & $\lambda_{1}$ & $\lambda_{i}$ & Corollary 16(1) & Lemma 28 & $\lambda_{2}$ & $\lambda_{i}$ & $\lambda_{j}$ & Corollary $16(2)$ & Lemma 38 \\
\hline$\lambda_{0}$ & $\lambda_{2}$ & $\lambda_{i}$ & Corollary 16(1) & Lemma 28 & $\lambda_{i}$ & $\lambda_{0}$ & $\lambda_{1}$ & Corollary $16(1)$ & Lemma 27 \\
\hline$\lambda_{0}$ & $\lambda_{i}$ & $\lambda_{j}$ & Corollary 16(2) & Lemma 38 & $\lambda_{i}$ & $\lambda_{0}$ & $\lambda_{2}$ & Corollary 16(1) & Lemma 27 \\
\hline$\lambda_{1}$ & $\lambda_{0}$ & $\lambda_{2}$ & Corollary 16(1) & Lemma 31 & $\lambda_{i}$ & $\lambda_{0}$ & $\lambda_{j}$ & Corollary $16(1)$ & Lemma 27 \\
\hline$\lambda_{1}$ & $\lambda_{0}$ & $\lambda_{i}$ & Corollary 16(1) & Lemma 30 & $\lambda_{i}$ & $\lambda_{1}$ & $\lambda_{2}$ & Corollary 16(1) & Lemma 27 \\
\hline$\lambda_{1}$ & $\lambda_{2}$ & $\lambda_{i}$ & Corollary 16(1) & Lemma 30 & $\lambda_{i}$ & $\lambda_{1}$ & $\lambda_{j}$ & Corollary $16(1)$ & Lemma 27 \\
\hline$\lambda_{1}$ & $\lambda_{i}$ & $\lambda_{j}$ & Corollary 16(1) & Lemma 30 & $\lambda_{i}$ & $\lambda_{2}$ & $\lambda_{j}$ & Corollary 16(1) & Lemma 28 \\
\hline$\lambda_{2}$ & $\lambda_{0}$ & $\lambda_{1}$ & - & - & $\lambda_{i}$ & $\lambda_{j}$ & $\lambda_{k}$ & Corollary $16(1)$ & Lemma 30 \\
\hline$\lambda_{2}$ & $\lambda_{0}$ & $\lambda_{i}$ & Corollary 16(1) & Lemma 27 & & & & & \\
\hline
\end{tabular}

Table 2: A summary of the argument in the case where $m>1$.

Suppose now that $m=1$. We argue exactly as for $m>1$, except in those cases where Lemma 28 is used. We consider these three cases separately, once again writing $\lambda_{i}$ for $\lambda_{i}^{(m, a)}$. When $\mu_{0}=\lambda_{0}, \mu_{1}=\lambda_{1}, \mu_{2}=\lambda_{i}$ with $i \geqslant 3$, we argue as in Proposition 21 case 3. When $\mu_{0}=\lambda_{0}, \mu_{1}=\lambda_{2}, \mu_{2}=\lambda_{i}$ with $i \geqslant 3$, we argue as in Proposition 21 case 4 . When $\mu_{0}=\lambda_{i}$ with $i \geqslant 3, \mu_{1}=\lambda_{2}, \mu_{2}=\lambda_{j}$ with $j \geqslant 3$, we have $\mu_{0}=$ $\frac{t_{n-1}}{y_{a_{i}}}\left(\frac{t_{n-1}}{y_{a}}+1\right), \mu_{1}=\left(\frac{t_{n-1}}{y_{a}}+1\right)^{2}$ and $\mu_{2}=\frac{t_{n-1}}{y_{a_{j}}}\left(\frac{t_{n-1}}{y_{a}}+1\right)$. Since $n \geqslant 5$ we can find $a_{k} \in\{0,1, \ldots, n-2\} \backslash\left\{a, a_{i}, a_{j}\right\}$. Let $p$ be a prime dividing $y_{a_{k}}$. Then $p$ divides $\mu_{0}$ and $\mu_{2}$ but $p$ does not divide $\mu_{1}$, contradicting Corollary 16(1).

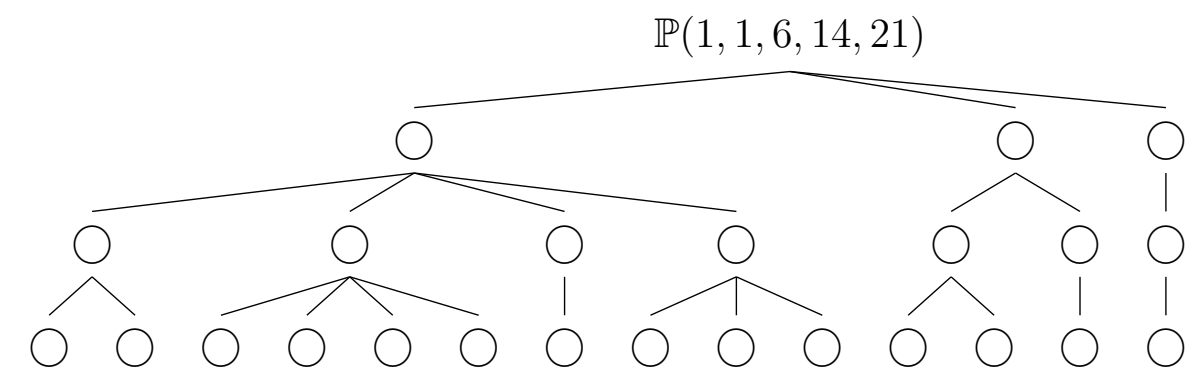

Figure 4: The graph of mutations between four-dimensional weighted projective spaces to a depth of 3

Remark 23. The requirement in Proposition 22 that $n \geqslant 5$ is necessary. Figure 4 shows the graph of mutations between four-dimensional weighted projective spaces starting from $X_{4}=\mathbb{P}(1,1,6,14,21)$ to a depth of three, where $\bigcirc$ denotes some weighted projective space. The rightmost branch of the tree above corresponds to a mutation with $\lambda_{0}=$ $1, \lambda_{1}=21$ and $\lambda_{2}=1$. It can be shown using the methods of $\S 7$ that this branch continues as a chain. We do not know what happens in the other branches at greater depth. 


\section{A The Sylvester Numbers and Related Sequences}

Recall the definition of Sylvester numbers from Definition 19. Recall also that $t_{n}:=y_{n}-1$.

Lemma 24. Let $n \geqslant 1$ and let $i \in\{0, \ldots, n-1\}$. Then $y_{i} \mid t_{n} / y_{i}+1$ and $y_{i} \backslash 2 t_{n} / y_{i}+1$.

Proof. We proceed by induction on $n$. The base case $n=1$ holds trivially. Note that:

$$
t_{n} / y_{i}+1=\left(t_{n-1} / y_{i}+1\right) y_{n-1}-\left(y_{n-1}-1\right)
$$

If $i \leqslant n-2$ then $y_{i} \mid y_{n-1}-1$ and, by the induction hypothesis, $y_{i} \mid t_{n-1} / y_{i}+1$. Thus $y_{i} \mid t_{n} / y_{i}+1$. If $i=n-1$ then $t_{n} / y_{i}+1=y_{n-1}$, which is certainly divisible by $y_{i}$. This completes the induction step, proving that $y_{i} \mid t_{n} / y_{i}+1$. It follows that $y_{i} \mid 2 t_{n} / y_{i}+2$, and so $y_{i} \backslash 2 t_{n} / y_{i}+1$.

Lemma 25. Let $\lambda_{i}^{(m, a)}$ be as in Proposition 21. For each $i \in\{0, \ldots, n\}$ the sequence $\left(\lambda_{i}^{(m, a)}\right)_{m \geqslant 0}$ is increasing.

Proof. This is a straightforward induction on $m$.

Lemma 26. Let $\lambda_{i}^{(m, a)}$ be as in Proposition 21. Fix $m \geqslant 0$. Then $\lambda_{0}^{(m, a)}, \lambda_{1}^{(m, a)}$, and $\lambda_{2}^{(m, a)}$ are pairwise coprime.

Proof. We begin by showing that $\lambda_{1}^{(m, a)}$ and $\lambda_{2}^{(m, a)}$ are coprime, proceeding by induction. The base case $m=0$ is trivial. Suppose that $\lambda_{1}^{(m-1, a)}$ and $\lambda_{2}^{(m-1, a)}$ are coprime, and suppose that there exists a prime $p$ dividing both $\lambda_{1}^{(m, a)}$ and $\lambda_{2}^{(m, a)}$. Then:

$$
p \mid \lambda_{1}^{(m-1, a)} \quad \text { and } \quad p \mid \frac{\left(\lambda_{1}^{(m-1, a)}+\lambda_{2}^{(m-1, a)}\right)^{2}}{\lambda_{0}^{(m-1, a)}}
$$

Thus $p \mid \lambda_{2}^{(m-1, a)}$, contradicting the hypothesis that $\lambda_{1}^{(m-1, a)}$ and $\lambda_{2}^{(m-1, a)}$ are coprime. This completes the induction step, showing that $\lambda_{1}^{(m, a)}$ and $\lambda_{2}^{(m, a)}$ are coprime for all $m$. It follows immediately that $\lambda_{0}^{(m, a)}=\lambda_{2}^{(m-1, a)}$ and $\lambda_{1}^{(m, a)}=\lambda_{1}^{(m-1, a)}$ are also coprime for all $m$.

Suppose now that $p \mid \lambda_{0}^{(m, a)}=\lambda_{2}^{(m-1, a)}$. Then $p \nmid \lambda_{1}^{(m, a)}$, as we have just seen, and so $p$ does not divide the numerator of

$$
\lambda_{2}^{(m, a)}=\frac{\left(\lambda_{1}^{(m, a)}+\lambda_{2}^{(m-1, a)}\right)^{2}}{\lambda_{0}^{(m-1, a)}}
$$

Thus $p \nmid \lambda_{2}^{(m, a)}$, so $\lambda_{0}^{(m, a)}$ and $\lambda_{2}^{(m, a)}$ are coprime.

Lemma 27. Let $\lambda_{i}^{(m, a)}$ be as in Proposition 21, let $m \geqslant 1$, and let $a \in\{0,1, \ldots, n-2\}$. There exists a prime $p$ such that $p \nmid \lambda_{0}^{(m, a)}, p \nmid \lambda_{1}^{(m, a)}$, and $p \mid \lambda_{i}^{(m, a)}$ for all $i \geqslant 2$. 
Proof. Lemma 25 implies that, for $m \geqslant 2, \lambda_{0}^{(m-1, a)}=\lambda_{2}^{(m-2, a)} \leqslant \lambda_{2}^{(m-1, a)}$. Thus

$$
\frac{\lambda_{1}^{(m, a)}+\lambda_{2}^{(m-1, a)}}{\lambda_{0}^{(m-1, a)}}>1
$$

for all $m \geqslant 1$, and so there is some prime $p$ such that $p \mid \lambda_{1}^{(m-1, a)}+\lambda_{2}^{(m-1, a)}$ but $p \nmid \lambda_{0}^{(m-1, a)}$. Thus $p \mid \lambda_{i}^{(m)}$ for all $i \in\{2, \ldots, n\}$. Lemma 26 now implies that $p \nmid \lambda_{0}^{(m, a)}$ and $p \nmid \lambda_{1}^{(m, a)}$.

Lemma 28. Let $\lambda_{i}^{(m, a)}$ be as in Proposition 21, let $m \geqslant 2$, and let $a \in\{0,1, \ldots, n-2\}$. There exists a prime $p$ such that $p \nmid \lambda_{1}^{(m, a)}, p \nmid \lambda_{2}^{(m, a)}$, and $p \mid \lambda_{i}^{(m, a)}$ for $i=0$ and all $i \geqslant 3$.

Proof. By Lemma 27 there exists a prime $p$ such that $p$ divides $\lambda_{i}^{(m-1, a)}$ for $i \in\{2, \ldots, n\}$ but $p$ does not divide $\lambda_{0}^{(m-1, a)}$ or $\lambda_{1}^{(m-1, a)}$. Thus $p \mid \lambda_{i}^{(m, a)}$ for all $i \in\{3, \ldots, n\}$ and, as $p \mid \lambda_{2}^{(m-1, a)}=\lambda_{0}^{(m, a)}$, we have by Lemma 26 that $p$ does not divide $\lambda_{1}^{(m, a)}$ or $\lambda_{2}^{(m, a)}$.

Lemma 29. Let $\lambda_{i}^{(m, a)}$ be as in Proposition 21, let $m \geqslant 0$, and let $a \in\{0,1, \ldots, n-2\}$. Then $2 \nmid \lambda_{0}^{(m, a)}, 2 \nmid \lambda_{2}^{(m, a)}$, and $2 \mid \lambda_{i}^{(m, a)}$ for $i=1$ and all $i \geqslant 3$.

Proof. This is a straightforward induction on $m$.

Lemma 30. Let $\lambda_{i}^{(m, a)}$ be as in Proposition 21, let $a \in\{0,1, \ldots, n-2\}$, and let $i \in$ $\{3, \ldots, n\}$. Then there exists $k>1$ such that for all $m \geqslant 0, k \backslash \lambda_{j}^{(m, a)}$ for $j \in\{0,2, i\}$ and $k \mid \lambda_{j}^{(m, a)}$ for $j \in\{0, \ldots, n\} \backslash\{0,2, i\}$.

Proof. We proceed by induction on $m$. For $m=0$ we have $\lambda_{j}^{(m, a)}=2 t_{n-1} / y_{a_{j}}$ for some $a_{j}$. If $y_{a_{i}} \neq 2$ then let $k$ be a prime dividing $y_{a_{i}}$; otherwise, let $k=4$. In either case $k$ has the desired properties and the claim holds.

Suppose now there exists $k>1$ such that $k \nmid \lambda_{j}^{(m-1, a)}$ for $j \in\{0,2, i\}$, and $k \mid \lambda_{j}^{(m-1, a)}$ for $j \in\{0, \ldots, n\} \backslash\{0,2, i\}$. Lemma 26 implies that $k$ and $\lambda_{0}^{(m-1)}$ are coprime. Thus as

$$
\lambda_{j}^{(m, a)}=\frac{\left.\lambda_{j}^{(m-1, a)}\left(\lambda_{1}^{(m-1, a}\right)+\lambda_{2}^{(m-1, a)}\right)}{\lambda_{0}^{(m-1, a)}} \quad \text { for } j \in\{3, \ldots, n\}
$$

we see that $k \mid \lambda_{j}^{(m, a)}$ for $j \in\{3, \ldots, n\} \backslash\{i\}$. Since $k \mid \lambda_{1}^{(m, a)}=\lambda_{1}^{(m-1, a)}$, by Lemma 26 again we have that $k \nmid \lambda_{0}^{(m, a)}$ and $k \nmid \lambda_{2}^{(m, a)}$. Finally as $k$ does not divide $\lambda_{2}^{(m-1, a)}$ but does divide $\lambda_{1}^{(m-1, a)}$, it does not divide $\lambda_{1}^{(m-1, a)}+\lambda_{2}^{(m-1, a)}$; since $k$ also does not divide $\lambda_{i}^{(m-1, a)}$, by the recursion formula it cannot divide $\lambda_{i}^{(m, a)}$ either.

Lemma 31. Let $\lambda_{i}^{(m, a)}$ be as in Proposition 21. There exists a prime $p$ such that, for $m \geqslant 0, p \mid \lambda_{1}^{(m, a)}$ and $p \nmid \lambda_{0}^{(m, a)}+\lambda_{2}^{(m, a)}$.

Proof. For the case $m=0$, take $p$ to be an odd prime dividing $\lambda_{1}=2 t_{n-1} / y_{a}$. Suppose there exists a prime $p$ such that $p \mid \lambda_{1}^{(m-1, a)}$ and $p \nmid \lambda_{0}^{(m-1, a)}+\lambda_{2}^{(m-1, a)}$. Then $p \nmid \lambda_{1}^{(m, a)}=$ $\lambda_{1}^{(m-1, a)}$ and and $p \mid \lambda_{1}^{(m-1, a)}\left(\lambda_{1}^{(m-1, a)}+2 \lambda_{2}^{(m-1, a)}\right)$. Now:

$$
\lambda_{0}^{(m, a)}+\lambda_{2}^{(m, a)}=\frac{\lambda_{2}^{(m-1, a)}\left(\lambda_{0}^{(m-1, a)}+\lambda_{2}^{(m-1, a)}\right)+\lambda_{1}^{(m-1, a)}\left(\lambda_{1}^{(m-1, a)}+2 \lambda_{2}^{(m-1, a)}\right)}{\lambda_{0}^{(m-1, a)}}
$$


and by Lemma 26, $p \nmid \lambda_{2}^{(m-1, a)}$. Thus $p \nmid \lambda_{0}^{(m)}+\lambda_{2}^{(m)}$. The result follows by induction on $m$.

Lemma 32. Let $\lambda_{i}^{(m, a)}$ be as in Proposition 21, let $b \in\{0,1, \ldots, n-2\}$ be such that $b \neq a$, and let $m \geqslant 0$. Then $y_{b} \mid \lambda_{2}^{(m, a)}-1$

Proof. The cases $m=0$ and $m=1$ are straightforward. Suppose now that $y_{b} \mid \lambda_{2}^{(m-2, a)}-1$ and $y_{b} \mid \lambda_{2}^{(m-1, a)}-1$. We have that:

$$
\lambda_{2}^{(m, a)}-1=\frac{\lambda_{1}^{(m-1, a)}\left(\lambda_{1}^{(m-1, a)}+2 \lambda_{2}^{(m-1, a)}\right)+\left(\lambda_{2}^{(m-1, a)}-1\right)\left(\lambda_{2}^{(m-1, a)}+\lambda_{0}^{(m-1, a)}\right)-\left(\lambda_{2}^{(m-2, a)}-1\right) \lambda_{2}^{(m-1, a)}}{\lambda_{0}^{(m-1, a)}}
$$

Since $y_{b} \mid 2 t_{n-1} / y_{a}=\lambda_{1}^{(m, a)}$, we have by Lemma 26 that $y_{b}$ and $\lambda_{0}^{(m-1, a)}$ are coprime. It thus suffices to show that $y_{b}$ divides the numerator of the above expression. But this holds by assumption. The Lemma follows by induction on $m$.

Definition 33. Let $X_{n}^{(m, a)}$ be as in $\S 7$ and let $h^{(m, a)}$ denote the sum of the weights of $X_{n}^{(m, a)}$.

Lemma 34.

$$
h^{(m, a)}= \begin{cases}2 t_{n-1} & \text { if } m=0 \\ \frac{\left(\lambda_{1}^{(m-1, a)}+\lambda_{2}^{(m-1, a)}\right) h^{(m-1, a)}}{\lambda_{0}^{(m-1, a)}} & \text { otherwise }\end{cases}
$$

and, for any $i \in\{3, \ldots, n\}$ :

$$
h^{(m, a)}=y_{k_{i}} \lambda_{i}^{(m, a)}
$$

Proof. This is a straightforward calculation.

Lemma 35. Let $\lambda_{i}^{(m, a)}$ be as in Proposition 21, let $a \in\{0,1, \ldots, n-2\}$, and let $m \geqslant 1$. Then:

$$
\frac{\lambda_{0}^{(m, a)} \lambda_{1}^{(m, a)}}{h^{(m, a)}}<\frac{1}{2 t_{n-1}}
$$

Proof. By Lemma 34 it suffices to prove that $\frac{2 t_{n-1} \lambda_{1}^{(m, a)}}{y_{k_{i}}}<\frac{\lambda_{i}^{(m, a)}}{\lambda_{0}^{(m, a)}}$. This evidently holds for $m=1$, and

$$
\frac{\lambda_{i}^{(m, a)}}{\lambda_{0}^{(m, a)}}=\frac{\lambda_{i}^{(m-1, a)}}{\lambda_{0}^{(m-1, a)}} \frac{\left(\lambda_{1}^{(m-1, a)}+\lambda_{2}^{(m-1, a)}\right)}{\lambda_{2}^{(m-1, a)}}>\frac{\lambda_{i}^{(m-1, a)}}{\lambda_{0}^{(m-1, a)}}
$$

The result follows by induction on $m$.

Lemma 36. Let $\lambda_{i}^{(m, a)}$ be as in Proposition 21, let $a \in\{0,1, \ldots, n-2\}$, let $m \geqslant 0$, and let $i \in\{3, \ldots, n\}$. Then:

$$
y_{k_{i}} \mid \lambda_{2}^{(m, a)}-\lambda_{0}^{(m, a)}
$$


Proof. We proceed by induction on $m$. The base case $m=0$ is trivial. Suppose now that $y_{k_{i}} \mid \lambda_{2}^{(m-1, a)}-\lambda_{0}^{(m-1, a)}$. Then, since $\lambda_{2}^{(m, a)}-\lambda_{0}^{(m, a)}=\left(\lambda_{2}^{(m, a)}-\lambda_{0}^{(m-1, a)}\right)-\left(\lambda_{2}^{(m-1, a)}-\lambda_{0}^{(m-1, a)}\right)$, it suffices to show that $y_{k_{i}}$ divides $\lambda_{2}^{(m, a)}-\lambda_{0}^{(m-1, a)}$. But:

$$
\begin{aligned}
& \lambda_{2}^{(m, a)}-\lambda_{0}^{(m-1, a)}= \\
& \frac{\lambda_{1}^{(m-1, a)}\left(\lambda_{1}^{(m-1, a)}+2 \lambda_{2}^{(m-1, a)}\right)+\left(\lambda_{2}^{(m-1, a)}-\lambda_{0}^{(m-1, a)}\right)\left(\lambda_{2}^{(m-1, a)}+\lambda_{0}^{(m-1, a)}\right)}{\lambda_{0}^{(m-1, a)}}
\end{aligned}
$$

Now $y_{k_{i}}$ divides $\lambda_{1}^{(m, a)}=\frac{2 t_{n-1}}{y_{a}}$ and $y_{k_{i}} \mid\left(\lambda_{2}^{(m-1, a)}-\lambda_{0}^{(m-1, a)}\right)$, so $y_{k_{i}}$ divides the numerator here. Lemma 26 implies that $y_{k_{i}}$ is coprime to the denominator. Thus $y_{k_{i}}$ divides $\lambda_{2}^{(m, a)}$ $\lambda_{0}^{(m-1, a)}$.

Lemma 37. Let $\lambda_{i}^{(m, a)}$ be as in Proposition 21, let $a \in\{0,1, \ldots, n-2\}$, let $m \geqslant 1$, and let $\kappa^{(m, a)}=h^{(m, a)}-\left(\lambda_{1}^{(m-1, a)}+\lambda_{2}^{(m-1, a)}\right)$. Then $\kappa^{(m, a)} \in\left\{2, \ldots, h^{(m, a)}-2\right\}$, and:

$$
\sum_{i=0}^{n}\left\{\frac{\lambda_{i}^{(m, a)} \kappa^{(m, a)}}{h^{(m, a)}}\right\}>n-1
$$

where $\{x\}$ denotes the fractional part of $x$.

Proof. The first statement follows immediately from Lemma 25. We claim that:

$$
\left\{\frac{\kappa^{(m, a)} \lambda_{0}^{(m, a)}}{h^{(m, a)}}\right\}= \begin{cases}1-\frac{1}{2 t_{n-1}} & \text { if } m \text { is odd } \\ 1-\frac{1}{2 t_{n-1}}-\frac{1}{y_{a}} & \text { if } m \text { is even }\end{cases}
$$

Note that:

$$
\begin{aligned}
\frac{\kappa^{(m, a)} \lambda_{0}^{(m, a)}}{h^{(m, a)}}= & 1-\frac{\left(\lambda_{1}^{(m-1, a)}+\lambda_{2}^{(m-1, a)}\right) \lambda_{0}^{(m, a)}}{y_{k_{i}} \lambda_{i}^{(m, a)}} \quad \text { by Lemma } 34 \\
= & 1-\frac{\lambda_{0}^{(m-1, a)} \lambda_{0}^{(m, a)}}{y_{k_{i}} \lambda_{i}^{(m-1, a)}} \\
= & 1-\frac{\lambda_{0}^{(m-2, a)} \lambda_{0}^{(m-1, a)} \lambda_{2}^{(m-1, a)}}{y_{k_{i}} \lambda_{i}^{(m-2, a)}\left(\lambda_{1}^{(m-2, a)}+\lambda_{2}^{(m-2, a)}\right)} \\
= & 1-\frac{\lambda_{2}^{(m-2, a)}\left(\lambda_{1}^{(m-2, a)}+\lambda_{2}^{(m-2, a)}\right)}{y_{k_{i}} \lambda_{i}^{(m-2, a)}} \\
= & 1-\frac{\lambda_{0}^{(m-2, a)}\left(\lambda_{1}^{(m-3, a)}+\lambda_{2}^{(m-3, a)}\right)}{y_{k_{i}} \lambda_{i}^{(m-2, a)}} \\
& \quad-\frac{\lambda_{2}^{(m-2, a)}\left(\lambda_{1}^{(m-2, a)}+\lambda_{2}^{(m-2, a)}\right)-\lambda_{0}^{(m-2, a)}\left(\lambda_{1}^{(m-2, a)}+\lambda_{0}^{(m-2, a)}\right)}{y_{k_{i}} \lambda_{i}^{(m-2, a)}}
\end{aligned}
$$


We claim that the last term (6) here is an integer. It is equal to:

$$
\frac{\left(\lambda_{2}^{(m-2, a)}-\lambda_{0}^{(m-2, a)}\right)\left(\lambda_{0}^{(m-2, a)}+\lambda_{1}^{(m-2, a)}+\lambda_{2}^{(m-2, a)}\right)}{h^{(m-2, a)}}
$$

Now:

$$
\begin{aligned}
\frac{\lambda_{0}^{(m-2, a)}+\lambda_{1}^{(m-2, a)}+\lambda_{2}^{(m-2, a)}}{h^{(m-2, a)}} & =\frac{h^{(m-2, a)}-\sum_{i=3}^{n} \lambda_{i}^{(m-2, a)}}{h^{(m-2, a)}} \\
& =1-\sum_{i=3}^{n} \frac{\lambda_{i}^{(m-2, a)}}{h^{(m-2, a)}} \\
& =1-\sum_{i=3}^{n} \frac{1}{y_{k_{i}}} \\
& =1-\sum_{i=0}^{n-2} \frac{1}{y_{i}}+\frac{1}{y_{a}} \\
& =\frac{1}{t_{n-1}}+\frac{1}{y_{a}}
\end{aligned}
$$

Hence (6) is equal to:

$$
\frac{\left(\lambda_{2}^{(m-2, a)}-\lambda_{0}^{(m-2, a)}\right)\left(t_{n-1} / y_{a}+1\right)}{t_{n-1}}
$$

Recall that $t_{n-1}=\prod_{i=0}^{n-2} y_{i}$. We will show that each $y_{i}$ divides the numerator of this expression. By Lemma 24 we have that $y_{a} \mid \frac{t_{n-1}}{y_{a}}+1$, and by Lemma 36 we have that, for all $i \neq a, y_{i} \mid \lambda_{2}^{(m-2, a)}-\lambda_{0}^{(m-2, a)}$. Hence (6) is an integer. Thus:

$$
\left\{\frac{\kappa^{(m, a)} \lambda_{0}^{(m, a)}}{h^{(m, a)}}\right\}=\left\{1-\frac{\lambda_{0}^{(m-2, a)}\left(\lambda_{1}^{(m-3, a)}+\lambda_{2}^{(m-3, a)}\right)}{y_{k_{i}} \lambda_{i}^{(m-2, a)}}\right\}=\left\{\frac{\kappa^{(m-2, a)} \lambda_{0}^{(m-2, a)}}{h^{(m-2, a)}}\right\}
$$

Since (5) holds for $m=1$ and $m=2$, by induction it holds for all $m$.

Lemma 34 implies that

$$
\frac{\kappa^{(m, a)}}{h^{(m, a)}}=1-\frac{\lambda_{0}^{(m-1, a)}}{h^{(m-1, a)}}
$$

We have:

$$
\begin{aligned}
\left\{\frac{\kappa^{(m, a)} \lambda_{1}^{(m, a)}}{h^{(m, a)}}\right\} & =\left\{1-\frac{\lambda_{0}^{(m-1, a)} \lambda_{1}^{(m, a)}}{h^{(m-1, a)}}\right\} \\
& =1-\frac{\lambda_{0}^{(m-1, a)} \lambda_{1}^{(m, a)}}{h^{(m-1, a)}}
\end{aligned}
$$


and for $i \in\{3, \ldots n\}$ we have:

$$
\begin{aligned}
\left\{\frac{\kappa^{(m, a)} \lambda_{i}^{(m, a)}}{h^{(m, a)}}\right\} & =\left\{1-\frac{\lambda_{i}^{(m-1, a)}\left(\lambda_{1}^{(m-1, a)}+\lambda_{2}^{(m-1, a)}\right)}{h^{(m-1, a)}}\right\} \\
& =\left\{1-\frac{\lambda_{1}^{(m-1, a)}+\lambda_{2}^{(m-1, a)}}{y_{k_{i}}}\right\} \\
& =\left\{1-\frac{\lambda_{1}^{(m-1, a)}}{y_{k_{i}}}-\frac{\lambda_{2}^{(m-1, a)}-1}{y_{k_{i}}}-\frac{1}{y_{k_{i}}}\right\} \\
& =1-\frac{1}{y_{k_{i}}}
\end{aligned}
$$

Putting this all together, for $m=1$ we obtain:

$$
\begin{aligned}
\sum_{i=0}^{n}\left\{\frac{\kappa^{(1, a)} \lambda_{i}^{(1, a)}}{\left.h^{(1, a)}\right\}}\right. & =\left\{\frac{\kappa^{(1, a)} \lambda_{2}^{(1, a)}}{h^{(1, a)}}\right\}+n-\sum_{i=0}^{n-2} \frac{1}{y_{i}}-\frac{1}{2 t_{n-1}} \\
& =\left\{\frac{\kappa^{(1)} \lambda_{2}^{(1)}}{h^{(1)}}\right\}+n-\frac{t_{n-1}-1}{t_{n-1}}-\frac{1}{2 t_{n-1}} \quad \text { by Lemma } 20 \\
& >n-1
\end{aligned}
$$

and for $m \geqslant 2$ we obtain:

$$
\begin{aligned}
\sum_{i=0}^{n}\left\{\frac{\kappa^{(m, a)} \lambda_{i}^{(m, a)}}{h^{(m, a)}}\right\} & \geqslant\left\{\frac{\kappa^{(m, a)} \lambda_{2}^{(m, a)}}{h^{(m, a)}}\right\}+n-\sum_{i=0}^{n-2} \frac{1}{y_{i}}-\frac{1}{2 t_{n-1}}-\frac{\lambda_{0}^{(m-1, a)} \lambda_{1}^{(m, a)}}{h^{(m-1, a)}} \\
& \geqslant(n-1)+\frac{1}{2 t_{n-1}}-\frac{\lambda_{0}^{(m-1, a)} \lambda_{1}^{(m, a)}}{h^{(m-1, a)}} \\
& >n-1
\end{aligned}
$$

where at the last step we used Lemma 35.

Lemma 38. Let $\lambda_{i}^{(m, a)}$ be as in Proposition 22, let $n \geqslant 5$, let $a \in\{0,1, \ldots, n-2\}$, and let $i, j \in\{3, \ldots, n\}$ be distinct. There exists a prime $p$ such that, for all $m \geqslant 0, p$ divides $\lambda_{1}^{(m, a)}, \lambda_{i}^{(m, a)}$, and $\lambda_{j}^{(m, a)}$ but $p$ does not divide $\lambda_{0}^{(m, a)}$ or $\lambda_{2}^{(m, a)}$.

Proof. We have $\lambda_{i}^{(0, a)}=\frac{t_{n-1}}{y_{k_{i}}}, \lambda_{j}^{(0, a)}=\frac{t_{n-1}}{y_{k_{j}}}$, and $\lambda_{1}^{(0, a)}=\frac{t_{n-1}}{y_{a}}$. Since $n \geqslant 5$ we can find $k_{l} \in\{0,1, \ldots, n-2\} \backslash\left\{a, k_{i}, k_{j}\right\}$. Let $p$ be a prime dividing $y_{k_{l}}$. Then $p$ divides $\lambda_{i}^{(0, a)}, \lambda_{j}^{(0, a)}$, and $\lambda_{1}^{(0, a)}$ and thus, by Lemma 26, $p$ divides neither $\lambda_{0}^{(0, a)}$ nor $\lambda_{2}^{(0, a)}$. We now proceed by induction on $m$ : the induction step is straightforward. 
Lemma 39. Let $\lambda_{i}^{(m, a)}$ be as in Proposition 22, let $a \in\{0,1, \ldots, n-2\}$, let $m \geqslant 1$, and let $\kappa^{(m, a)}=h^{(m, a)}-h^{(m-1, a)}$. Then $\kappa^{(m, a)} \in\left\{2, \ldots, h^{(m, a)}-2\right\}$, and:

$$
\sum_{i=0}^{n}\left\{\frac{\lambda_{i}^{(m, a)} \kappa^{(m, a)}}{h^{(m, a)}}\right\}<2
$$

where $\{x\}$ denotes the fractional part of $x$.

Proof. The first statement follows immediately from Lemma 25. The conclusions of Lemma 34 hold here too, and thus:

$$
\frac{\kappa^{(m, a)}}{h^{(m, a)}}=1-\frac{\lambda_{0}^{(m-1, a)}}{\lambda_{1}^{(m-1, a)}+\lambda_{2}^{(m-1, a)}}
$$

It follows that:

$$
\left\{\frac{\kappa^{(m, a)} \lambda_{2}^{(m, a)}}{h^{(m, a)}}\right\}=\left\{-\lambda_{2}^{(m, a)} \frac{\lambda_{0}^{(m-1, a)}}{\lambda_{1}^{(m-1, a)}+\lambda_{2}^{(m-1, a)}}\right\}=0
$$

and, for $i \in\{3, \ldots, n\}$ :

$$
\left\{\frac{\kappa^{(m, a)} \lambda_{i}^{(m, a)}}{h^{(m, a)}}\right\}=\left\{-\lambda_{i}^{(m, a)} \frac{\lambda_{0}^{(m-1, a)}}{\lambda_{1}^{(m-1, a)}+\lambda_{2}^{(m-1, a)}}\right\}=0
$$

Thus:

$$
\sum_{i=0}^{n}\left\{\frac{\kappa^{(m, a)} \lambda_{i}^{(m, a)}}{h^{(m, a)}}\right\}=\left\{\frac{\kappa^{(m, a)} \lambda_{0}^{(m, a)}}{h^{(m, a)}}\right\}+\left\{\frac{\kappa^{(m, a)} \lambda_{1}^{(m, a)}}{h^{(m, a)}}\right\}<2
$$

\section{Acknowledgements}

The authors thank Mohammad Akhtar and Alessio Corti for useful conversations. This research is supported by the Royal Society, ERC Starting Investigator Grant number 240123, the Leverhulme Trust, and EPSRC grant EP/I008128/1.

\section{References}

[1] Mohammad Akhtar, Tom Coates, Sergey Galkin, and Alexander M. Kasprzyk. Minkowski polynomials and mutations. SIGMA Symmetry Integrability Geom. Methods Appl., 8:094, pp. 707, 2012.

[2] Mohammad Akhtar and Alexander M. Kasprzyk. Mutations of fake weighted projective planes. To appear in Proc. Edinburgh Math. Soc. (2). arXiv:1302.1152, 2013. 
[3] Denis Auroux. Mirror symmetry and $T$-duality in the complement of an anticanonical divisor. J. Gökova Geom. Topol. GGT, 1:51-91, 2007.

[4] Gennadiy Averkov, Jan Krümpelmann, and Benjamin Nill. Largest integral simplices with one interior integral point: Solution of Hensley's conjecture and related results. arXiv:1309.7967, 2013.

[5] Victor V. Batyrev. Toric degenerations of Fano varieties and constructing mirror manifolds. In The Fano Conference, pages 109-122. Univ. Torino, Turin, 2004.

[6] Victor V. Batyrev, Ionuţ Ciocan-Fontanine, Bumsig Kim, and Duco van Straten. Conifold transitions and mirror symmetry for Calabi-Yau complete intersections in Grassmannians. Nuclear Phys. B, 514(3):640-666, 1998.

[7] Victor V. Batyrev, Ionut Ciocan-Fontanine, Bumsig Kim, and Duco van Straten. Mirror symmetry and toric degenerations of partial flag manifolds. Acta Math., 184(1):1-39, 2000.

[8] A. A. Borisov and L. A. Borisov. Singular toric Fano three-folds. Mat. Sb., 183(2):134-141, 1992. text in Russian. English transl.: Russian Acad. Sci. Sb. Math., 75 (1993), 277-283.

[9] Weronika Buczyńska. Fake weighted projective spaces. arXiv:0805.1211v1, 2008.

[10] Tom Coates, Alessio Corti, Sergey Galkin, Vasily Golyshev, and Alexander Kasprzyk. Mirror symmetry and Fano manifolds. In European Congress of Mathematics Kraków, 2-7 July, 2012, pages 285-300, 2014.

[11] Tom Coates, Alessio Corti, Sergey Galkin, and Alexander M. Kasprzyk. Quantum periods for 3-dimensional Fano manifolds. arXiv:1303.3288, 2013.

[12] Heinke Conrads. Weighted projective spaces and reflexive simplices. Manuscripta Math., 107(2):215-227, 2002.

[13] V. I. Danilov. The geometry of toric varieties. Uspekhi Mat. Nauk, 33(2(200)):85-134, $247,1978$.

[14] Tohru Eguchi, Kentaro Hori, and Chuan-Sheng Xiong. Gravitational quantum cohomology. Internat. J. Modern Phys. A, 12(9):1743-1782, 1997.

[15] Sergey Fomin and Andrei Zelevinsky. Cluster algebras. I. Foundations. J. Amer. Math. Soc., 15(2):497-529 (electronic), 2002.

[16] Sergey Galkin and Alexandr Usnich. Mutations of potentials. Preprint IPMU 100100, 2010.

[17] Mark Gross, Paul Hacking, and Sean Keel. Birational geometry of cluster algebras. arXiv: 1309. 2573, 2013.

[18] Kentaro Hori and Cumrun Vafa. Mirror symmetry. arXiv:hep-th/0002222v3, 2000.

[19] A. R. Iano-Fletcher. Working with weighted complete intersections. In Explicit birational geometry of 3-folds, volume 281 of London Math. Soc. Lecture Note Ser., pages 101-173. Cambridge Univ. Press, Cambridge, 2000. 
[20] Nathan Owen Ilten. Mutations of Laurent polynomials and flat families with toric fibers. SIGMA Symmetry Integrability Geom. Methods Appl., 8:47-53, 2012.

[21] Alexander M. Kasprzyk. Bounds on fake weighted projective space. Kodai Math. J., 32(2):197-208, 2009.

[22] Alexander M. Kasprzyk. Canonical toric Fano threefolds. Canad. J. Math., 62(6):1293-1309, 2010.

[23] Alexander M. Kasprzyk. Classifying terminal weighted projective space. arXiv:1304.3029, 2013.

[24] Alexander M. Kasprzyk and Benjamin Nill. Fano polytopes. In Anton Rebhan, Ludmil Katzarkov, Johanna Knapp, Radoslav Rashkov, and Emanuel Scheidegger, editors, Strings, Gauge Fields, and the Geometry Behind - the Legacy of Maximilian Kreuzer, pages 349-364. World Scientific, 2012.

[25] Benjamin Nill. Volume and lattice points of reflexive simplices. Discrete and Computational Geometry, 37:301-320, 2007.

[26] Yu. G. Prokhorov. The degree of Fano threefolds with canonical Gorenstein singularities. Mat. Sb., 196(1):81-122, 2005.

[27] Miles Reid. Canonical 3-folds. Journéees de Géeometrie Algéebrique d'Angers, pages 273-310, 1980.

[28] Miles Reid. Minimal models of canonical 3-folds. In Algebraic varieties and analytic varieties (Tokyo, 1981), volume 1 of Adv. Stud. Pure Math., pages 131-180. NorthHolland, Amsterdam, 1983.

[29] Miles Reid. Young person's guide to canonical singularities. Algebraic Geometry, 1:345-414, 1987.

[30] G. C. Shephard. Decomposable convex polyhedra. Mathematika, 10:89-95, 1963.

[31] J. J. Sylvester. On a point in the theory of vulgar fractions. American Journal of Mathematics, 3(4):332-335, 1880. 